\title{
Electromechanical Models of Ferroelectric Materials
}

\author{
J.E. Huber \\ Department of Engineering Science, \\ University of Oxford, Parks Rd. Oxford, OX1 3PJ.
}

\begin{abstract}
Models of the electro-mechanical behaviour of ferroelectric materials are reviewed. Starting from the constitutive relationships for piezoelectrics and estimates of the response of piezoelectric composites, the development of models is traced from the macroscale through to the micro-scale. Derivations of models based on extensions of classical plasticity and crystal plasticity theory are given, following the literature, and example applications of these models are shown. The formation of domain patterns is discussed and minimum energy methods based on the concept of compatibility are used to derive typical domain patterns for tetragonal and rhombohedral ferroelectrics. Methods for modelling the evolution of domain patterns are described. Finally the outlook for future directions in modelling of ferroelectrics is discussed.
\end{abstract}

\section{Introduction}

Among the ferroic functional materials, the ferroelectrics are distinguished by the presence of a spontaneous dipole moment or polarization that can be reversed through the action of electric field. Typical ferroelectrics are noncentrosymmetric crystalline materials, such as the perovskite oxide alloys in the lead zirconate titanate system, barium titanate and others. They exhibit strong coupling between electrical and mechanical effects and this brought them to prominence through diverse applications of the piezoelectric effect in sonar, acoustics and later ultrasound and precision actuators. There is an extensive literature introducing the materials and their physics (Lines and Glass (1977); Xu (1991); Sidorkin (2006); Tagantsev et al. (2010)). During the last 30 years interest in the ferroelectrics intensified following the growth of technological opportunities: increasing use of automated control has driven demand for sensors and actuators based on the piezoelectric principle. More recently the ability to produce ferroelectrics in a wide 
variety of forms such as thin films, fibres, micro-machined structures and nano-particles has opened up further opportunities.

While the study of the physics of ferroelectrics has been ongoing for about a century, the advent of practical constitutive models, that can be used to predict the material performance for engineering design purposes, is relatively recent. From the constitutive modelling perspective, the main variables of interest are electrical (electric field/electric displacement), mechanical (stress/strain) and thermal (temperature/entropy). Thermal effects are central to the processing and preparation of ferroelectrics, which commonly have a symmetry breaking phase transition between a high temperature non-ferroelectric phase and a low temperature ferroelectric phase that is key to the appearance of spontaneous polarization during processing. The influence of temperature on polarization through the pyroelectric effect, and on the dielectric permittivity, are also of importance. The wellknown phenomonology of Devonshire (1949) captured the dominant effects and this phenomenological approach to modelling remains a key tool for understanding ferroelectrics. However, from the point of view of engineering design, stable isothermal conditions are often desirable, and can often be maintained by suitable thermal controls. In this case, sensitivity of the material behaviour to temperature, and the generation of heat, are still essential considerations, but the key variables for constitutive modelling become the electrical and mechanical variables. In this spirit, the present article reviews constitutive models of ferroelectrics that relate electric field/electric displacement and stress/strain under isothermal conditions.

With the development of devices that have complex structures such as the composite pillar arrangements of sonar transducers and the embedded electrodes used in multi-layer stack actuators, a requirement arises for models that can handle multi-axiality of the field variables. Equally the ability to model non-linearity in the constitutive response due to the intense fields generated near defects, or geometric features has become important. The chapter is structured as an introduction to several modelling approaches, starting with macroscopic models, proceeding to finer scales by considering the effects of ceramic grains and domains, and eventually looking at recent research on the arrangements of domains and domain walls. The focus is on relatively simple models that can be applied to a wide range of materials, but examples are given to illustrate the application to common ferroelectric systems. 


\section{Origins of Ferroelectricity and Piezoelectricity}

The origin of spontaneous polarization and the capability for that polarization to be switched between distinct states can be understood by first considering the crystal structure of a typical ferroelectric. Taking the perovskite oxide $\mathrm{PbTiO}_{3}$ example, the arrangement of a unit cell in the tetragonal phase is shown in figure 1 . This contains an offset central $\mathrm{Ti}^{4+}$, which combined with offsets of other ions and displacement of their charges produces a net polarization aligned with the $c$-axis of the unit cell. An applied stress produces small displacements of the ions giving rise to the usual elastic effects, but can also modify the charge distribution, changing the dipole moment of the cell by the piezoelectric effect. Because of the lack of a centre of symmetry, an applied stress can change the dipole moment. Similarly, an applied electric field will produce linear dielectric effects but can also distort the unit cell, resulting in the converse piezoelectric effect.

The linear, reversible effects can be described by a free energy function of strain $\epsilon_{i j}$ and electric displacement $D_{i}$ written in the form:

$$
\Psi(\boldsymbol{\epsilon}, \boldsymbol{D})=\frac{1}{2} \epsilon_{i j} c_{i j k l}^{D} \epsilon_{k l}-D_{k} h_{k i j} \epsilon_{i j}+\frac{1}{2} D_{i} \beta_{i k}^{\epsilon} D_{k}
$$

Defining stress $\sigma_{i j}$ as $\partial \Psi / \partial \epsilon_{i j}$ and electric field $E_{i}$ as $\partial \Psi / \partial D_{i}$ gives the constitutive relations

$$
\begin{aligned}
\sigma_{i j} & =c_{i j k l}^{D} \epsilon_{k l}-h_{k i j} D_{k} \\
E_{i} & =-h_{i k l} \epsilon_{k l}+\beta_{i k}^{\epsilon} D_{k}
\end{aligned}
$$

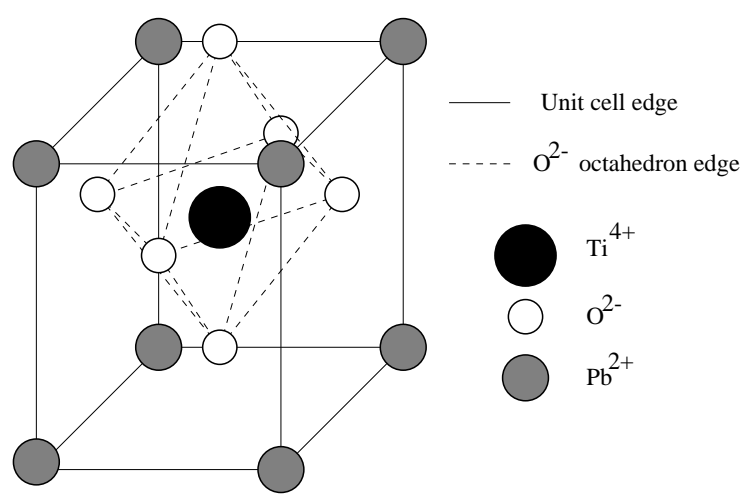

Figure 1. Unit cell of tetragonal $\mathrm{PbTiO}_{3}$. 
where $c_{i j k l}^{D}$ is the tensor of elastic moduli, $h_{k i j}$ are piezoelectric coefficients, and $\beta_{i k}^{\epsilon}$ is the tensor of dielectric impermeabilities. The superscript $D$ indicates that the elastic moduli are those measured with the electric displacement held constant - that is, in open circuit conditions. Similarly, superscript $\epsilon$ indicates that the dielectric impermeabilities are those measured with the strain held constant - that is, with clamped boundary conditions. In (2) and (3) the strain and electric displacement are to be understood as variables measured relative to a reference state that is free of macroscopic stress and electric field. Practical measurements can be achieved by observations of surface displacement and changes in the state of surface charge on a material sample.

For later use, it is worth noting that (2) and (3) can be rearranged and inverted into various forms. Making $\epsilon_{i j}$ and $D_{i}$ the subjects yields equations of the form:

$$
\begin{aligned}
\epsilon_{i j} & =s_{i j k l}^{E} \sigma_{k l}+d_{k i j} E_{k} \\
D_{i} & =d_{i k l} \sigma_{k l}+\varepsilon_{i k}^{\sigma} E_{k}
\end{aligned}
$$

Two further rearrangements are commonly used:

$$
\begin{aligned}
\epsilon_{i j} & =s_{i j k l}^{D} \sigma_{k l}+g_{k i j} D_{k} \\
E_{i} & =-g_{i k l} \sigma_{k l}+\beta_{i k}^{\sigma} D_{k}
\end{aligned}
$$

and

$$
\begin{aligned}
\sigma_{i j} & =c_{i j k l}^{E} \epsilon_{k l}-e_{k i j} E_{k} \\
D_{i} & =e_{i k l} \epsilon_{k l}+\varepsilon_{i k}^{\epsilon} E_{k}
\end{aligned}
$$

Equations (2)-(9) will be used interchangeably, with expressions relating the coefficients given where needed.

When a sufficiently strong electric field is applied to the material, ferroelectric switching can occur, reversing the polarization as shown in figure 2 . The stable offset of the $\mathrm{Ti}^{4+}$ cation from the centre of the unit cell in tetragonal lead titanate is towards one face of the cell. There are six such stable states in polar tetragonal crystals with the corresponding spontaneous polarizations

$$
\boldsymbol{P}= \pm P_{0}\left(\begin{array}{l}
1 \\
0 \\
0
\end{array}\right), \pm P_{0}\left(\begin{array}{l}
0 \\
1 \\
0
\end{array}\right) \text { and } \pm P_{0}\left(\begin{array}{l}
0 \\
0 \\
1
\end{array}\right)
$$

in Cartesian co-ordinates aligned to the unit cell edges. Here $P_{0}$ is the magnitude of the spontaneous polarization. 


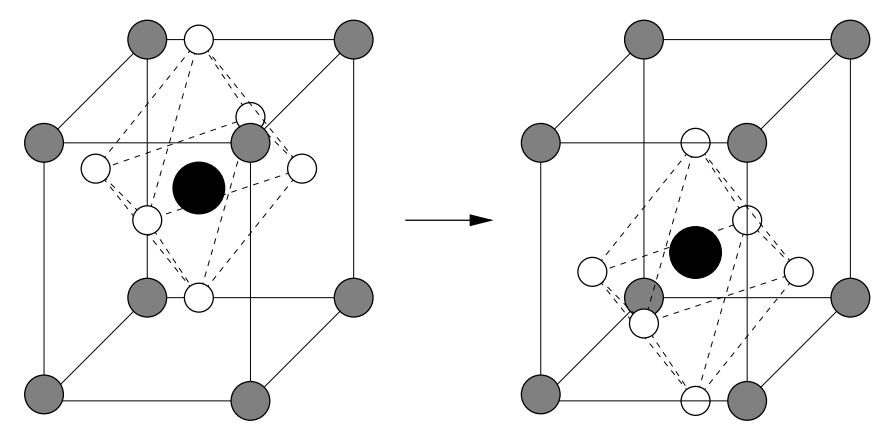

Figure 2. Ferroelectric switching in the unit cell of tetragonal $\mathrm{PbTiO}_{3}$.

The existence of the set of six polarization states in polar tetragonal crystals allows two distinct kinds of ferroelectric switching: one in which the polarization vector reverses in sign, or turns through $180^{\circ}$ and a second in which the polarization vector turns through $90^{\circ}$. Meanwhile, the corresponding spontaneous strain states of the tetragonal unit cell are given by

$$
\epsilon_{i j}=\frac{3}{2} \epsilon_{0}\left(p_{i} p_{j}-\frac{1}{3} \delta_{i j}\right)
$$

where $\boldsymbol{p}=\boldsymbol{P} / P_{0}, \delta_{i j}$ is the Kronecker delta, and $\epsilon_{0}$ is a measure of the spontaneous strain magnitude. It follows from (11) that $180^{\circ}$ switching, induces no net change in spontaneous strain. However, $90^{\circ}$ switching results in a change in both spontaneous polarization and strain. The term ferroelastic is used to describe $90^{\circ}$ switching when driven by an applied stress.

A ferroelectric crystal typically contains regions of uniform polarization, or domains, separated by thin boundaries (domain walls). Each domain has a state of spontaneous strain and polarization that can be changed by an electric field. Hence the overall state of polarization and strain of a ferroelectric crystal can change. Effectively, the macroscopic material stores a state of remanent strain $\boldsymbol{\epsilon}^{r}$ and remanent polarization $\boldsymbol{P}^{r}$. By "remanent" it is meant that the strain and polarization remain after macroscopic electric field and stress are removed. Changing these remanent quantities through the application of stress or electric field can also affect the elastic, piezoelectric and dielectric response. To illustrate this point, take as an example the tetragonal crystal system, where the microscopic piezoelectric 
response of a single domain is governed by the coefficients

$$
\begin{aligned}
h_{k i j}=h_{33} p_{k} p_{i} p_{j}+h_{31}\left(p_{k} \delta_{i j}-p_{k} p_{i} p_{j}\right) \\
+h_{15}\left(\delta_{k i} p_{j}+\delta_{k j} p_{i}-2 p_{k} p_{i} p_{j}\right)
\end{aligned}
$$

$h_{33}, h_{31}$ and $h_{15}$ being material constants. Then $180^{\circ}$ switching converts $\boldsymbol{p}$ to $-\boldsymbol{p}$ and since $h_{k i j}$ is an odd function of $\boldsymbol{p}$ its sign is also reversed. The $h_{k i j}$ coefficients are also changed by $90^{\circ}$ switching. By contrast the dielectric and elastic coefficients, $\beta_{i j}^{\epsilon}$ and $c_{i j k l}^{D}$, are even functions of $\boldsymbol{p}$ and are unchanged by $180^{\circ}$ switching. However, they can be changed by $90^{\circ}$ switching.

In order to model the effects of ferroelectric switching, the free energy expression (1) requires modification. This can be achieved by the introduction of higher-order terms to capture non-linearity. The existence of several stable states then leads to a multi-well energy structure that has been used in classical models of ferroelectrics (Devonshire (1949)) and can be used to simulate the formation of ferroelectric domains (Li et al. (2001); Wang et al. (2004); Su and Landis (2007)). In typical ferroelectrics at equilibrium, however, it is observed that almost all of the material is in a state at or close to the bottom of one of the energy wells. The energy wells are deep, resulting in thin domain walls (typically only a few lattice parameters across) and high barriers to switching. This motivates modelling approaches analogous to the constrained theory of martensites wherein each material point is assumed to be in a state close to the bottom of an energy well (Ball and James (1987); Shu and Bhattacharya (2001)), with a locally convex free energy.

Taking ferroelectric switching into account, (2) and (3) can be rewritten to represent the response of the ferroelectric in the form:

$$
\begin{aligned}
\sigma_{i j} & =c_{i j k l}^{D}\left(\boldsymbol{\epsilon}^{r}, \boldsymbol{P}^{r}\right)\left(\epsilon_{k l}-\epsilon_{k l}^{r}\right)-h_{k i j}\left(\boldsymbol{\epsilon}^{r}, \boldsymbol{P}^{r}\right)\left(D_{k}-P_{k}^{r}\right) \\
E_{i} & =-h_{i k l}\left(\boldsymbol{\epsilon}^{r}, \boldsymbol{P}^{\boldsymbol{r}}\right)\left(\epsilon_{k l}-\epsilon_{k l}^{r}\right)+\beta_{i k}^{\epsilon}\left(\boldsymbol{\epsilon}^{r}, \boldsymbol{P}^{r}\right)\left(D_{k}-P_{k}^{r}\right)
\end{aligned}
$$

where the dependency of elastic, dielectric and piezoelectric coefficients on the state of remanent strain and polarization has been made explicit. In (13) and (14) the strain and electric displacement are referred to a stress and electric field free state with $\left(\boldsymbol{\epsilon}^{r}, \boldsymbol{P}^{\boldsymbol{r}}\right)=0$. While (13) and (14) treat $\boldsymbol{\epsilon}^{\boldsymbol{r}}$ and $\boldsymbol{P}^{r}$ as state variables, there is no reason to suppose that these variables together contain sufficient information to specify the internal state of the material, or even to specify the coefficients in equations 13 and 14. Treating the two quantities $\left(\boldsymbol{\epsilon}^{\boldsymbol{r}}, \boldsymbol{P}^{\boldsymbol{r}}\right)$ as state variables is also inconvenient from a practical measurement perspective in that the readily available measurements typically indicate only $(\boldsymbol{\epsilon}, \boldsymbol{D})$, and then only by their changes. 
Nevertheless, the remanent strain and polarization have been widely used as experimental indicators of the switching process (Lynch (1996); Liu and Huber (2007)), and adopted as internal state variables in models of switching (Hwang et al. (1995); Kamlah and Tsakmakis (1999); Landis (2002); Huber and Fleck (2001)).

\section{Piezoelectric Composites}

Taking ( 2) and (3) as a starting point, it is of interest to consider composites whose components exhibit piezoelectric behaviour. In the first instance, the small signal material response is considered, comprising elastic, dielectric and piezoelectric behaviour, connecting together the stress $\sigma_{i j}$, strain $\epsilon_{i j}$, electric field $E_{i}$ and electric displacement $D_{i}$. The strain is taken to be the symmetrized gradient of a continuous displacement field $\boldsymbol{u}$ :

$$
\epsilon_{i j}=\frac{1}{2}\left(u_{i, j}+u_{j, i}\right)
$$

Analogously, electrostatic conditions are assumed such that the electric field can be derived from a scalar potential, $\phi$ :

$$
E_{i}=-\phi_{, i}
$$

Mechanical and electrical equilibrium equations can be written as

$$
\begin{aligned}
\sigma_{i j, j} & =0 \\
D_{i, i} & =0
\end{aligned}
$$

where it is assumed that no body forces act and no free charge is present. At internal interfaces, the absence of body forces and charges implies continuity of the normal components of stress and electric displacement:

$$
\begin{aligned}
\Delta \sigma_{i j} n_{j} & =0 \\
\Delta D_{i} n_{i} & =0
\end{aligned}
$$

where $\Delta$ indicates the jump in each quantity at the interface and $\boldsymbol{n}$ is the interface normal direction. At external free surfaces, the normal component of stress is the surface traction $t_{i}$ and the jump in normal component of electric displacement is the surface charge density $q$ :

$$
\begin{aligned}
\sigma_{i j} n_{j} & =t_{i} \\
\Delta D_{i} n_{i} & =q
\end{aligned}
$$


Since the permittivity of ferroelectric materials is typically $2-3$ orders of magnitude greater than that of air or vacuum, the approximation $D_{i} n_{i}=-q$ at free surfaces can often be made with good accuracy. Conditions similar to (19) and (20) apply to the strain and electric field if the displacement and electric potential are continuous at internal interfaces. Let $s$ be a vector tangent to an interface across which the strain and electric field jump. Then

$$
\begin{aligned}
\Delta \epsilon_{i j} s_{j} & =0 \\
\Delta E_{i} s_{i} & =0
\end{aligned}
$$

It is convenient to group the constitutive equations together into a matrix form:

$$
\left(\begin{array}{c}
\sigma_{i j} \\
E_{i}
\end{array}\right)=\left(\begin{array}{cc}
c_{i j k l}^{D} & -h_{k i j} \\
-h_{i k l} & \beta_{i k}^{\epsilon}
\end{array}\right)\left(\begin{array}{c}
\epsilon_{k l} \\
D_{k}
\end{array}\right)
$$

and note that, when dealing with a linear piezoelectric material, any spontaneous strain or polarization are taken to provide a constant ground state, relative to which the strain and electric displacement are measured. Likewise, the material state is assumed unchanged by any loading applied, such that the material coefficients or moduli are constants.

The constitutive relation (25) can be thought of as describing the macroscopic behaviour of a bulk material, or (with suitable coefficients) the behaviour of a microscopic region of material. In relating the microscopic to the macroscopic electromechanical response it is convenient to think of the bulk material as a composite comprising various constituent regions, with differing material coefficients. In a polycrystalline ferroelectric, the regions may represent differently oriented ceramic grains, or at a finer scale, individual ferroelectric domains. The composite could also contain nonpiezoelectric components $\left(h_{k i j}=0\right)$ but all constituent regions are assumed to be solid, insulating dielectrics. For a compact notation, rewrite (25) as

$$
\sigma=C \epsilon
$$

identifying bold symbols with collections of tensor quantities:

$$
\boldsymbol{\sigma}=\left(\begin{array}{c}
\sigma_{i j} \\
E_{i}
\end{array}\right) ; \boldsymbol{C}=\left(\begin{array}{cc}
c_{i j k l}^{D} & -h_{k i j} \\
-h_{i k l} & \beta_{i k}^{\epsilon}
\end{array}\right) ; \boldsymbol{\epsilon}=\left(\begin{array}{c}
\epsilon_{k l} \\
D_{k}
\end{array}\right)
$$

Taking (26) to represent the microscopic, or local, constitutive relation, a corresponding macroscopic relationship may be written for a bulk region of material, assumed sufficiently large and statistically homogenous that it may be treated as an effective medium:

$$
\bar{\sigma}=C^{o} \bar{\epsilon}
$$


Here, the notation $\boldsymbol{\bullet}=\frac{1}{V} \int \bullet d V$ has been used to indicate volume averages and the macroscopic variables $\overline{\boldsymbol{\sigma}}$ and $\overline{\boldsymbol{\epsilon}}$ are identified averages of their microscopic counterparts. This step is justified by considering a macroscopic region subject to a charge distribution on its boundary given by $-\tilde{D}_{i} n_{i}$ where $n_{i}$ is the outward surface normal. Then the average of the microscopic electric displacement $D_{i}(\boldsymbol{x})$ is given by

$$
\begin{aligned}
\bar{D}_{i} & =\frac{1}{V} \int_{V} D_{i} d V=\frac{1}{V} \int_{V} D_{j} x_{i, j} d V=\frac{1}{V} \int_{V}\left(D_{j} x_{i}\right), j-D_{j, j} x_{i} d V \\
& =\frac{1}{V} \int_{S} D_{j} n_{j} x_{i} d S=\frac{\tilde{D}_{j}}{V} \int_{S} x_{i} n_{j} d S=\frac{\tilde{D}_{j}}{V} \int_{V} x_{i, j} d V=\tilde{D}_{i}
\end{aligned}
$$

Electrical equilibrium (18) has been used along with Gauss' theorem to convert between volume and surface integrals. Also note that $x_{i, j}=\delta_{i j}$, the Kronecker delta. An analogous argument holds for the averaging of the stress field, using (17), and similar arguments apply to the averaging of electric field and strain: consider the macroscopic region having electric potential $\phi=-\tilde{E}_{i} x_{i}$ on its boundary. Then

$$
\begin{aligned}
\bar{E}_{i} & =\frac{1}{V} \int_{V} E_{i} d V=\frac{1}{V} \int_{V}-\phi_{, i} d V=\frac{1}{V} \int_{S}-\phi n_{i} d S \\
& =\frac{1}{V} \int_{S} \tilde{E}_{j} x_{j} n_{i} d S=\frac{\tilde{E}_{j}}{V} \int_{S} x_{i} n_{j} d S=\frac{\tilde{E}_{j}}{V} \int_{V} x_{i, j} d V=\tilde{E}_{i}
\end{aligned}
$$

where (16) has been used.

In general, finding the effective properties $\boldsymbol{C}^{\boldsymbol{o}}$ from a known spatial distribution of $\boldsymbol{C}$ is a homogenization problem dependent on the detailed arrangement of the components of the composite. Various bounds (Bisegna and Luciano (1996)) and estimates (Dunn and Taya (1993)) for $\boldsymbol{C}^{\boldsymbol{o}}$ have been developed for piezoelectric composites. Here, the self-consistent theory of composites, originally derived by by Hill (1965) is described, as this furnishes a practical means of estimating $\boldsymbol{C}^{\boldsymbol{o}}$ for certain common arrangements of composite that can be approximated as a matrix material containing ellipsoidal inclusions. The self-consistent theory makes the approximation that each component of the composite behaves as if it were an ellipsoidal inclusion embedded in a surrounding matrix with properties $C^{o}$. Then the difference between the local electromechanical fields, by Eshelby's argument (Eshelby (1957)) uniform within the inclusion, and the corresponding mean fields is given by

$$
\sigma-\bar{\sigma}=-C^{\star}(\epsilon-\bar{\epsilon})
$$


Where

$$
C^{\star}=C^{o}\left(\mathcal{S}^{-1}-I\right)
$$

In (32), $\boldsymbol{I}$ represents the collection of identity tensors given by

$$
\boldsymbol{I}=\left(\begin{array}{cc}
\frac{1}{2}\left(\delta_{i k} \delta_{j l}+\delta_{j k} \delta_{i l}\right) & 0 \\
0 & \delta_{i k}
\end{array}\right)
$$

and $\mathcal{S}$ is the collection of Eshelby tensors relating a notional unconstrained transformation strain and electric displacement, say $\boldsymbol{\epsilon}^{t}$, of an inclusion in a medium of moduli $\boldsymbol{C}^{\boldsymbol{o}}$ to the constrained strain and electric displacement of the inclusion. That is, $\boldsymbol{\epsilon}=\mathcal{S} \boldsymbol{\epsilon}^{t}$.

For particular combinations of the symmetries of $\boldsymbol{C}^{\boldsymbol{o}}$ and the ellipsoidal inclusion, explicit formulae for the components of $\mathcal{S}$ are known (Dunn and Taya (1993)). In the most general case, the piezoelectric Eshelby tensors may be obtained by numerical integration (Huber et al. (1999)). The inversion of a collection of tensors such as $\mathcal{S}$ or $\boldsymbol{C}$ is interpreted in the sense that if $\boldsymbol{\sigma}=\boldsymbol{C} \boldsymbol{\epsilon}$ then $\boldsymbol{\epsilon}=\boldsymbol{C}^{-1} \boldsymbol{\sigma}$. This inversion may be accomplished by unpacking the elements of $\boldsymbol{C}$ into a matrix form and using matrix inversion methods.

Making use of (26), (28) and (31) gives

$$
\epsilon=\left(C+C^{\star}\right)^{-1}\left(C^{o}+C^{\star}\right) \bar{\epsilon}=A \bar{\epsilon}
$$

which defines $\boldsymbol{A}$ as the set of concentration tensors for strain and electric displacement, specific to a particular ellipsoidal component of the composite. Averaging over the volume of the composite shows that $\overline{\boldsymbol{A}}=\boldsymbol{I}$. Equally, since for each component of the composite $\boldsymbol{\sigma}=\boldsymbol{C} \boldsymbol{\epsilon}$, then

$$
\bar{\sigma}=\overline{C A} \bar{\epsilon}
$$

and the effective properties $\boldsymbol{C}^{\boldsymbol{o}}$ can be identified as the volume average $\overline{\boldsymbol{C} \boldsymbol{A}}$.

The estimate of the effective moduli provided by (35) is in implicit form since the concentration tensors $\boldsymbol{A}$ depend on the effective moduli themselves through equation 32 ; iterative recalculation of $\boldsymbol{C}^{\boldsymbol{o}}$ and $\boldsymbol{C}^{\star}$ normally gives rapid convergence. Alternative approaches to the estimation of effective properties include unit cell or representative volume element calculations (Kouznetsova et al. (2001)), bounds (Bisegna and Luciano (1996)) and asymptotic homogenization (Kanoute et al. (2009)). The self-consistent homogenization has the advantage of lying within the wellknown Hashin-Shtrikman bounds in cases such as that of elastic spherical inclusions. Also, when there is strong contrast in moduli between the components of the composite the rigorous bounds are typically far apart, while 
Table 1. Material data for a piezoelectric composite.

\begin{tabular}{cccccc}
\hline & Epoxy & PZT & & Epoxy & PZT \\
\hline$c_{1111}^{D}(\mathrm{GPa})$ & 8.0 & 157 & $\beta_{11}^{\epsilon}\left(\mathrm{m} \mathrm{nF}^{-1}\right)$ & 26.9 & 0.246 \\
$c_{3333}^{D}(\mathrm{GPa})$ & 8.0 & 175 & $\beta_{33}^{\epsilon}\left(\mathrm{m} \mathrm{nF}^{-1}\right)$ & 26.9 & 0.481 \\
$c_{2323}^{D}(\mathrm{GPa})$ & 1.8 & 47.2 & $h_{311}\left(\mathrm{GV} \mathrm{m}^{-1}\right)$ & 0 & -1.02 \\
$c_{1212}^{D}(\mathrm{GPa})$ & 1.8 & 35.8 & $h_{333}\left(\mathrm{GV} \mathrm{m}^{-1}\right)$ & 0 & 4.58 \\
$c_{1122}^{D}(\mathrm{GPa})$ & 4.4 & 85.4 & $h_{113}\left(\mathrm{GV} \mathrm{m}^{-1}\right)$ & 0 & 2.30 \\
$c_{1133}^{D}(\mathrm{GPa})$ & 4.4 & 73.0 & & & \\
\hline
\end{tabular}

the self-consistent method can still give useful estimates provided that any extremely stiff or compliant phases are dilute. However, in certain cases of strong contrast such as porous composites with significant volume fraction of porosity, the self-consistent estimate becomes inaccurate.

\subsection{Example of a piezoelectric composite}

The self- consistent estimate of $\boldsymbol{C}^{\boldsymbol{o}}$ can provide insight into the practical design of piezoelectric composites for engineering applications. An example is the piezoelectric hydrophone, used in sonar detection. Of key importance in this application is the piezoelectric response to hydrostatic stress. Rearrangement of (25) gives

$$
\left(\begin{array}{c}
\epsilon_{i j} \\
E_{i}
\end{array}\right)=\left(\begin{array}{cc}
s_{i j k l}^{D} & g_{k i j} \\
-g_{i k l} & \beta_{i k}^{\sigma}
\end{array}\right)\left(\begin{array}{c}
\sigma_{k l} \\
D_{k}
\end{array}\right)
$$

where $s_{i j k l}^{D}=\left(c^{D}\right)_{i j k l}^{-1}, g_{k i j}=\left(c^{D}\right)_{i j p q}^{-1} h_{k p q}$ and $\beta_{i k}^{\sigma}=\beta_{i k}^{\epsilon}-h_{i p q} s_{p q r s}^{D} h_{k r s}$. Taking the axis of piezoelectric poling as the $x_{3}$ axis, then the open circuit voltage generated under hydrostatic pressure, which determines the sensitivity of the hydrophone, is governed by the piezoelectric coefficient $g_{3 k k}$, often given the symbol $g_{h}$. Typical bulk ferroelectrics have $g_{333}$ coefficients of opposite sign to $g_{311}$ with $g_{311} \approx-g_{333} / 2$, resulting in low values of $g_{h}$. However, by constructing a composite of ferroelectric fibres or pillars aligned with the $x_{3}$ axis and embedded in a compliant matrix, significant enhancements in the macroscopic $g_{h}$ value can be achieved. To illustrate this an example is calculated using material data taken from Bisegna and Luciano (1996), see table 1. This allows a direct comparison between self-consistent estimates and the variational bounds computed therein. The composite considered has a matrix of isotropic epoxy resin and contains round fibres of PZT-7A, taken to have transverse isotropy.

Figure 3 shows self-consistent estimates of the dielectric impermeabilities $\beta_{11}^{\epsilon}$ and $\beta_{33}^{\epsilon}$ for this composite. To approximate fibres aligned with the $x_{3}$ 

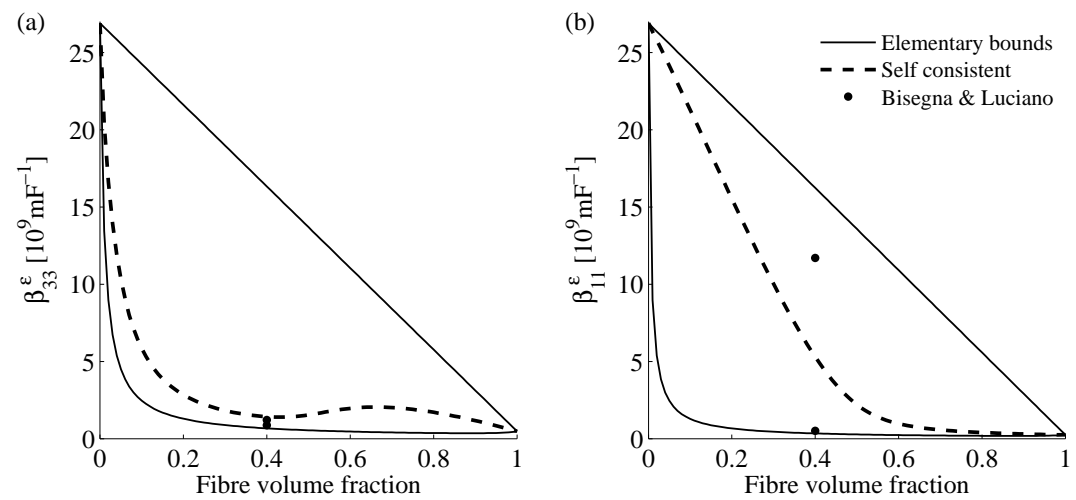

Figure 3. Self-consistent estimates of impermeabilities (a) $\beta_{33}^{\epsilon}$ and (b) $\beta_{11}^{\epsilon}$ for a piezoelectric fibre composite.

axis, ellipsoidal inclusions with minor radii $a=b$ and major radius $c=10 a$ were used in the calculation, the $c$ axis being aligned with $x_{3}$. The volume fraction $f$ of fibres is varied in the range $0-1$. Elementary bounds on $\beta_{11}^{\epsilon}$ and $\beta_{33}^{\epsilon}$ of the Voigt type $\left(\boldsymbol{C}^{\boldsymbol{V}}=\overline{\boldsymbol{C}}\right)$ and Reuss type $\left(\boldsymbol{C}^{\boldsymbol{R}}=\left(\overline{\boldsymbol{C}^{-1}}\right)^{-1}\right)$ are also shown, along with tighter variational bounds derived by Bisegna and Luciano at $f=0.4$. Note that the elementary bounds are far apart in this case; improved variational bounds can be much closer, as in figure 3a, but can still show a wide range as in figure $3 \mathrm{~b}$. The self-consistent estimates give an indication of how the properties of the composite change with volume fraction, and agree well with the bounds. In particular figure 3 shows that $\beta_{33}^{\epsilon}$ is greatly reduced by adding a small volume of fibres, while $\beta_{11}^{\epsilon}$ remains fairly high. This may be expected because the fibres provide connectivity in the $x_{3}$ direction, such that the electric field component $E_{3}$ is similar in the fibres and matrix, while the absence of fibre connectivity in the $x_{1}$ direction causes the $E_{1}$ component of electric field to be concentrated in the matrix material. Similar anisotropy is seen in the elastic properties of the composite.

A consequence of this strong anisotropy can be seen in the hydrostatic voltage coefficient $g_{h}$; self- consistent estimates of $g_{h}^{o}$ for the composite, nor- 


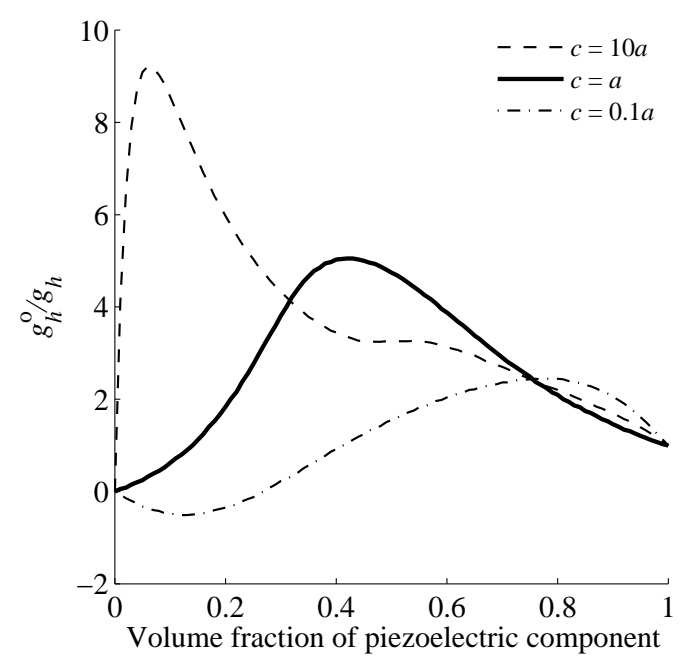

Figure 4. Self-consistent estimates of enhancement to the hydrostatic voltage coefficient $g_{h}$ in a piezoelectric fibre composite.

malized by the $g_{h}$ coefficient of the piezoelectric component of the composite are shown in figure 4. Here, various aspect ratios for the piezoelectric component are compared. In a fibrous composite $(c=10 a)$, an order of magnitude enhancement of $g_{h}$ can be achieved by using a low volume fraction of fibres. The enhancement is mainly due to the elastic anisotropy of the composite. A particulate composite $(c=a)$ shows less enhancement of $g_{h}^{o}$ and requires a high volume fraction of piezoelectric particles to achieve optimum enhancement, while disc-like piezoelectric particles $c=0.1 a$ are even less effective.

\section{Models of Ferroelectric Switching}

Now consider an extension of the linear piezoelectric constitutive laws (25) to represent material states with a remanent polarization and strain:

$$
\left(\begin{array}{c}
\sigma_{i j} \\
E_{i}
\end{array}\right)=\left(\begin{array}{cc}
c_{i j k l}^{D} & -h_{k i j} \\
-h_{i k l} & \beta_{i k}^{\epsilon}
\end{array}\right)\left(\begin{array}{c}
\epsilon_{k l}-\epsilon_{k l}^{r} \\
D_{k}-P_{k}^{r}
\end{array}\right)
$$

Or, in the compact notation of section 3 ,

$$
\sigma=C\left(\epsilon-\epsilon^{r}\right)
$$


Changes in $\epsilon^{\boldsymbol{r}}$ are somewhat analogous to increments of plastic strain in the classical theory of plasticity; there are two significant differences: The first is that here $\boldsymbol{C}$ is dependent on $\boldsymbol{\epsilon}^{\boldsymbol{r}}$; thus changes in $\boldsymbol{C}$ must be included in the theory. The second is that, unlike classical plasticity, where plastic straining can proceed indefinitely through the nucleation and motion of dislocations, the remanent strain and polarization of ferroelectricity are limited in range. This is because no further ferroelectric switching can happen once all of the material has switched. Thus the $P_{0}$ and $\epsilon_{0}$ of (10) and (11) place limits on $\boldsymbol{\epsilon}^{\boldsymbol{r}}$. In plasticity theory such a limit on strain could be handled through strain-dependent hardening rules, and similar methods are adopted in the case of ferroelectrics. Finally it should be noted that $\boldsymbol{\epsilon}^{\boldsymbol{r}}$ itself consists of both a remanent strain and a remanent polarization part, but this added complication has no impact on the theoretical development, except for making the expressions rather cumbersome if written out in full.

A goal of constitutive modelling is to provide tangent moduli $\boldsymbol{C}^{\boldsymbol{t}}$ to relate increments, or rates of applied loading to the incremental material response such that

$$
\dot{\sigma}=C^{t} \dot{\boldsymbol{\epsilon}}
$$

In sections 4.1 and 4.2 models of ferroelectric switching are described that are analogous to classical plasticity and crystal plasticity respectively, following closely the corresponding developments in the literature (Bassiouny et al. (1988); Cocks and McMeeking (1999); Huber et al. (1999); Huber and Fleck (2001); Kamlah and Tsakmakis (1999); Landis (2002)).

\subsection{Classical plasticity model}

In the spirit of classical plasticity, a constitutive framework for the ferroelectric material can be developed by postulating a free energy expression similar to (1) but including a dissipative part associated with switching (Cocks and McMeeking (1999)):

$$
\Psi=\frac{1}{2}\left(\epsilon-\epsilon^{r}\right) \boldsymbol{C}\left(\boldsymbol{\epsilon}-\epsilon^{\boldsymbol{r}}\right)+\Psi^{r}\left(\epsilon^{\boldsymbol{r}}\right)
$$

As in the linear case, the stress and electric field can be derived from the free energy.

$$
\boldsymbol{\sigma}=\frac{\partial \Psi}{\partial \boldsymbol{\epsilon}}
$$

Then the second law of thermodynamics requires that the rate of work done by external agencies acting on the material, $\boldsymbol{\sigma} \dot{\boldsymbol{\epsilon}}$, is at least as great as the rate of increase in free energy, or

$$
\sigma \dot{\epsilon}-\dot{\Psi} \geq 0
$$


The free energy rate is

$$
\dot{\Psi}=\boldsymbol{\sigma} \dot{\boldsymbol{\epsilon}}-\boldsymbol{\sigma} \dot{\epsilon}^{r}+\frac{\partial \Psi^{r}}{\partial \boldsymbol{\epsilon}^{r}} \dot{\boldsymbol{\epsilon}}^{r}+\frac{\partial \Psi}{\partial \boldsymbol{C}} \frac{\partial \boldsymbol{C}}{\partial \boldsymbol{\epsilon}^{r}} \dot{\boldsymbol{\epsilon}}^{r}
$$

where the final term is due to the dependence of the elastic, dielectric and piezoelectric moduli on the remanent strain and polarization state. Equally, by defining

and

$$
\boldsymbol{\sigma}^{B}=\frac{\partial \Psi^{r}}{\partial \boldsymbol{\epsilon}^{r}}+\frac{\partial \Psi}{\partial \boldsymbol{C}} \frac{\partial \boldsymbol{C}}{\partial \boldsymbol{\epsilon}^{r}}
$$

$$
\hat{\sigma}=\sigma-\sigma^{B}
$$

the free energy rate can be written as

$$
\dot{\Psi}=\sigma \dot{\epsilon}-\hat{\sigma} \dot{\epsilon}^{r}
$$

and (42) can then be expressed as

$$
\hat{\sigma} \dot{\epsilon}^{r} \geq 0
$$

One way to satisfy (47) is for there to be a convex surface in $\hat{\sigma}$ space enclosing the origin such that switching occurs only at states of $\hat{\boldsymbol{\sigma}}$ that lie on the surface, and such that the associated rates $\dot{\boldsymbol{\epsilon}}^{r}$ are directed towards the outward normal of the surface at point $\hat{\sigma}$. This is analogous to the convex yield surface and associated flow of classical plasticity. Continuing the development in this way, let switching occur only when

$$
G\left(\hat{\boldsymbol{\sigma}}, \boldsymbol{\epsilon}^{\boldsymbol{r}}\right)=0
$$

and the rates $\dot{\boldsymbol{\epsilon}}^{r}$ be defined by

$$
\dot{\boldsymbol{\epsilon}}^{r}=\dot{\lambda} \frac{\partial G}{\partial \hat{\sigma}}
$$

where $\dot{\lambda}$ is analogous to a plastic multiplier. Then, since $\dot{G}=0$ during switching,

$$
\frac{\partial G}{\partial \hat{\sigma}} \dot{\hat{\sigma}}+\frac{\partial G}{\partial \epsilon^{r}} \dot{\boldsymbol{\epsilon}}^{r}=0
$$

So far, the value of $\dot{\lambda}$ is arbitrary and the determination of the rate of switching corresponding to a given loading rate requires additional information. This can be specified through an analogue of the hardening rules found in classical plasticity, in this case connecting increments in the "back" fields $\sigma^{B}$ to the increments of the remanent quantities $\epsilon_{i j}^{r}$ and $P_{i}^{r}$. Let

$$
\dot{\sigma}^{B}=H \dot{\epsilon}^{r}
$$


where $\boldsymbol{H}$ is a collection of tensors that depend on the state of the material and can be derived from a specification of $\Psi$, or defined directly as an alternative to specifying $\Psi$. Proper specification of the switching surface $G$ and hardening rule, through $\boldsymbol{H}$, will define the non-linear response of the material. The requirement to set $G$ convex motivates the use of relatively simple quadratic expressions for the switching surface (Cocks and McMeeking (1999); Huber and Fleck (2001); Landis (2002)), placing the majority of the richness in the material behaviour into $\boldsymbol{H}$. Using (51) and (50) the rates $\dot{\lambda}$ can be found. First it is convenient to define $\boldsymbol{n}=\partial G / \partial \hat{\boldsymbol{\sigma}}$ and $\boldsymbol{m}=\partial G / \partial \boldsymbol{\epsilon}^{r}$. Then

$$
\boldsymbol{n} \dot{\boldsymbol{\sigma}}=\boldsymbol{n} \dot{\boldsymbol{\sigma}}^{B}-\boldsymbol{m} \dot{\boldsymbol{\epsilon}}^{r}=\dot{\lambda}(\boldsymbol{n} \boldsymbol{H} \boldsymbol{n}-\boldsymbol{m n})
$$

The rates $\dot{\lambda}$ during switching are given by

$$
\dot{\lambda}=\frac{n}{n H n-m n} \dot{\sigma}
$$

and thus the rates of the remanent quantities are given by

$$
\dot{\epsilon}^{r}=\Lambda \dot{\sigma}
$$

where

$$
\Lambda=\frac{n \otimes n}{n H n-m n}
$$

Finally, using (38), (39) and (54) the tangent moduli can be derived:

$$
\begin{aligned}
\dot{\boldsymbol{\sigma}}=\boldsymbol{C}^{t} \dot{\boldsymbol{\epsilon}} & =\dot{\boldsymbol{C}}\left(\boldsymbol{\epsilon}-\boldsymbol{\epsilon}^{r}\right)+\boldsymbol{C} \dot{\boldsymbol{\epsilon}}-\boldsymbol{C} \dot{\boldsymbol{\epsilon}}^{r} \\
& =\left(\frac{\partial \boldsymbol{C}}{\partial \epsilon^{r}} \boldsymbol{C}^{-1} \boldsymbol{\sigma}-\boldsymbol{C}\right) \boldsymbol{\Lambda} \dot{\boldsymbol{\sigma}}+\boldsymbol{C} \dot{\boldsymbol{\epsilon}}
\end{aligned}
$$

where the inverse of $\boldsymbol{C}$ represents a set of tensors of the form

$$
\boldsymbol{C}^{-1}=\left(\begin{array}{cc}
s_{i j k l}^{E} & d_{k i j} \\
d_{i k l} & \varepsilon_{i k}^{\sigma}
\end{array}\right)
$$

dependent on the current material state. Rearranging (56) gives

$$
\dot{\boldsymbol{\sigma}}=\left(\boldsymbol{I}+\boldsymbol{C} \boldsymbol{\Lambda}-\frac{\partial \boldsymbol{C}}{\partial \boldsymbol{\epsilon}^{r}} \boldsymbol{C}^{-1} \boldsymbol{\sigma} \boldsymbol{\Lambda}\right)^{-1} \boldsymbol{C} \dot{\boldsymbol{\epsilon}}
$$

and the tangent moduli are identified as

$$
C^{t}=\left(\boldsymbol{I}+C \boldsymbol{\Lambda}-\frac{\partial \boldsymbol{C}}{\partial \epsilon^{r}} \boldsymbol{C}^{-1} \boldsymbol{\sigma} \boldsymbol{\Lambda}\right)^{-1} \boldsymbol{C}
$$


Care is needed in interpreting the term $\left(\partial \boldsymbol{C} / \partial \boldsymbol{\epsilon}^{\boldsymbol{r}}\right) \boldsymbol{C}^{-1} \boldsymbol{\sigma} \boldsymbol{\Lambda}$ - this consists of a collection of tensors of the form

$$
\frac{1}{\boldsymbol{n H n - \boldsymbol { m n }}}\left(\begin{array}{cc}
T_{i j k l}^{\prime \prime} & T_{k i j}^{\prime} \\
T_{i k l}^{\prime} & T_{i k}
\end{array}\right)
$$

which, if written out in full, produce lengthy expressions such as

$$
\begin{aligned}
T_{i j k l}^{\prime \prime}= & \left(\frac{\partial c_{i j r s}^{D}}{\partial \epsilon_{p q}^{r}} \frac{\partial G}{\partial \hat{\sigma}_{p q}}+\frac{\partial c_{i j r s}^{D}}{\partial P_{q}^{r}} \frac{\partial G}{\partial \hat{E}_{q}}\right)\left(s_{r s m n}^{E} \sigma_{m n}+d_{t r s} E_{t}\right) \frac{\partial G}{\partial \hat{\sigma}_{k l}} \\
& -\left(\frac{\partial h_{r i j}}{\partial \epsilon_{p q}^{r}} \frac{\partial G}{\partial \hat{\sigma}_{p q}}+\frac{\partial h_{r i j}}{\partial P_{q}^{r}} \frac{\partial G}{\partial \hat{E}_{q}}\right)\left(d_{r m n} \sigma_{m n}+\varepsilon_{r t}^{\sigma} E_{t}\right) \frac{\partial G}{\partial \hat{\sigma}_{k l}}
\end{aligned}
$$

and so forth. Note that a consequence of the presence of these terms in the tangent moduli is that the tangent moduli depend not only on the material state, represented through the remanent quantities in $\boldsymbol{\epsilon}^{r}$, but also on the applied stress and electric field loading $\boldsymbol{\sigma}$. The physical effect captured by this term is the energy released or absorbed by changes in the material compliance or permittivity due to switching. In most practical ferroelectrics this effect is small because the magnitude of the reversible strain and electric displacement represented by $\boldsymbol{C}^{-1} \boldsymbol{\sigma}$ is much less than the magnitude of the remanent quantities $\boldsymbol{\epsilon}^{r}$, while the compliances change only fractionally during switching.

Models of the type derived in this section have been illustrated by Huber and Fleck (2001) and Landis (2002). The models have the advantage of simplicity, which enables them to be incorporated readily into finite element codes for engineering design calculations. However, there are disadvantages. Apart from the difficulty of specifying hardening rules that accurately capture both the switching process and the eventual saturation of switching, the main objection to the model leading to (59) is perhaps the assumption that the remanent strain and polarization are sufficient as internal variables to capture the material state. Experimental evidence (Liu and Lynch (2006); Liu and Huber (2007)) certainly suggests that the material behaviour is more complex. This motivates models with a greater number of internal variables that build up a macroscopic picture of material behaviour from an understanding of the microscopic process of switching, as explored in the following section.

\subsection{Crystal plasticity model}

In the context of crystal plasticity, the starting point is consideration of a single crystal and the microscopic processes that lead to changes in the 
macroscopic $\boldsymbol{\epsilon}^{\boldsymbol{r}}$ of that crystal. The switching process of a ferroelectric crystal is modelled by replacing the slip systems of classical plasticity with a set of transformation systems that correspond to the motion of domain walls. The macroscopic switching effect arises from a number of these transformation systems acting incrementally and simultaneously. The development here closely follows that in the literature (Huber et al. (1999); Huber and Fleck (2004); Pathak and McMeeking (2008)).

First consider a single crystal or grain within a ceramic, in which there are several domains with distinct, symmetry related orientations of the polar axis. In section 2 it was shown that there are six symmetry related variants in the polar tetragonal crystal system. More generally, let there be $n$ variants such that the $I$ th variant has spontaneous strain $\epsilon_{i j}^{I}$ and polarization $P_{i}^{I}$, the two quantities being represented in compact notation by $\boldsymbol{\epsilon}^{I}$. Similarly, let each variant have moduli $\boldsymbol{C}^{I}$ with inverse $\boldsymbol{S}^{I}$ and occupy volume fraction $v^{I}$ of the crystal. Define $\boldsymbol{\epsilon}$ and $\boldsymbol{\sigma}$ to represent effective strain/electric displacement and stress/electric field values at the macroscopic level of the entire crystal. Similarly, let the local values in individual domains be $\boldsymbol{\epsilon}^{l}, \boldsymbol{\sigma}^{l}$. Then, by the arguments in (29) and (30), $\boldsymbol{\epsilon}=\overline{\boldsymbol{\epsilon}^{l}}$ and $\boldsymbol{\sigma}=\overline{\boldsymbol{\sigma}^{l}}$. But these arguments do not allow $\boldsymbol{\epsilon}^{\boldsymbol{r}}$ to be immediately identified with the volume average $\overline{\boldsymbol{\epsilon}^{I}}=\sum v^{I} \boldsymbol{\epsilon}^{I}$. Instead the constitutive law for the crystal can be written

$$
\epsilon-\epsilon^{r}=S \sigma
$$

where $\boldsymbol{S}$ is the inverse of $\boldsymbol{C}$, the set of macroscopic moduli for the crystal. At the microscopic level, each material point has the constitutive relation

$$
\boldsymbol{\epsilon}^{l}-\boldsymbol{\epsilon}^{I}=\boldsymbol{S}^{I} \boldsymbol{\sigma}^{l}
$$

Averaging (63) gives

$$
\boldsymbol{\epsilon}-\overline{\boldsymbol{\epsilon}^{I}}=\overline{\boldsymbol{S}^{I} \boldsymbol{\sigma}^{l}}
$$

Now, if the assumption is made that the stress and electric field are uniform within the crystal, then $\boldsymbol{\sigma}^{l}=\boldsymbol{\sigma}$ and

$$
\epsilon-\overline{\epsilon^{I}}=\overline{S^{I}} \boldsymbol{\sigma}
$$

so that it is then possible to identify $\boldsymbol{S}=\overline{\boldsymbol{S}^{I}}$ and $\boldsymbol{\epsilon}^{r}=\overline{\boldsymbol{\epsilon}^{I}}$. This is the Reuss approximation at the level of the single crystal or grain. Proceeding on this basis, define a set of transformations $\alpha:=I \rightarrow J$ that correspond to the actions of moving domain walls in transforming material of crystal variant $I$ into material of crystal variant $J$. Let $\dot{f}^{\alpha}$ be the rate of volume fraction transformation by $\alpha$. Then

$$
\dot{\boldsymbol{\epsilon}}^{r}=\dot{\overline{\boldsymbol{\epsilon}^{I}}}=\sum_{\alpha} \dot{f}^{\alpha} \boldsymbol{\Delta} \boldsymbol{\epsilon}^{\alpha}
$$


where $\Delta \boldsymbol{\epsilon}^{\alpha}=\boldsymbol{\epsilon}^{J}-\boldsymbol{\epsilon}^{I}$. Similarly, defining $\Delta \boldsymbol{S}^{\alpha}=\boldsymbol{S}^{J}-\boldsymbol{S}^{I}$ gives

$$
\dot{\boldsymbol{S}}=\dot{\overline{\boldsymbol{S}^{I}}}=\sum_{\alpha} \dot{f}^{\alpha} \boldsymbol{\Delta} \boldsymbol{S}^{\alpha}
$$

Considering energy, the electromechanical work stored in the crystal by virtue of its elastic, dielectric and piezoelectric moduli is

$$
w=\frac{1}{2} \sigma \boldsymbol{S} \boldsymbol{\sigma}
$$

Now suppose that each transformation system dissipates energy of at least $G^{\alpha c}$ per unit volume in converting a volume of material from variant $I$ to variant $J$. The second law of thermodynamics requires that

$$
\boldsymbol{\sigma} \dot{\boldsymbol{\epsilon}}-\dot{w}-\sum_{\alpha} \dot{f}^{\alpha} G^{\alpha c} \geq 0
$$

Noting, from (62), that

$$
\sigma \dot{\epsilon}=\sigma S \dot{\sigma}+\sigma \dot{S} \sigma+\sigma \dot{\epsilon}^{r}
$$

and, from (68),

$$
\dot{w}=\sigma \boldsymbol{S} \dot{\boldsymbol{\sigma}}+\frac{1}{2} \sigma \dot{\boldsymbol{S}} \boldsymbol{\sigma}
$$

allows (69) to be written as

$$
\sum_{\alpha} \dot{f}^{\alpha} G^{\alpha}-\sum_{\alpha} \dot{f}^{\alpha} G^{\alpha c} \geq 0
$$

where $G^{\alpha}$ is the thermodynamic driving force for switching, given by

$$
G^{\alpha}=\sigma \Delta \epsilon^{\alpha}+\frac{1}{2} \sigma \Delta S^{\alpha} \sigma
$$

A criterion for ferroelectric switching that satisfies (72) is that switching occurs only when

$$
G^{\alpha}=G^{\alpha c} \text { and } \dot{f}^{\alpha}>0
$$

Following classical crystal plasticity, the set of tangent moduli for the crystal can be found by connecting increments in $G^{\alpha}$ to the increments $\dot{f}^{\alpha}$ via a hardening rule. For simplicity, consider independent hardening, with

$$
\dot{G}^{\alpha c}=h^{\alpha} \dot{f}^{\alpha}
$$

Then, from (73)

$$
\dot{G}^{\alpha}=\dot{\sigma} \hat{\epsilon}^{\alpha}
$$


where

$$
\hat{\boldsymbol{\epsilon}}^{\alpha}=\Delta \boldsymbol{\epsilon}^{\alpha}+\Delta S^{\alpha} \boldsymbol{\sigma}
$$

and since during switching $\dot{G}^{\alpha}=\dot{G}^{\alpha c}$

$$
\dot{f}^{\alpha}=\frac{1}{h^{\alpha}} \hat{\boldsymbol{\epsilon}}^{\alpha} \dot{\boldsymbol{\sigma}}
$$

The incremental constitutive relation derived from (62) is

$$
\dot{\boldsymbol{\epsilon}}=\boldsymbol{S} \dot{\boldsymbol{\sigma}}+\dot{\boldsymbol{S}} \boldsymbol{\sigma}+\dot{\boldsymbol{\epsilon}}^{r}
$$

or, making use of (77),

$$
\dot{\boldsymbol{\epsilon}}=\boldsymbol{S} \dot{\boldsymbol{\sigma}}+\sum_{\alpha} \dot{f}^{\alpha} \hat{\boldsymbol{\epsilon}}^{\alpha}
$$

Substituting from (78) gives

$$
\dot{\boldsymbol{\epsilon}}=\left(\boldsymbol{S}+\sum_{\alpha} \frac{1}{h^{\alpha}} \hat{\boldsymbol{\epsilon}}^{\alpha} \otimes \hat{\boldsymbol{\epsilon}}^{\alpha}\right) \dot{\boldsymbol{\sigma}}
$$

and the tangent moduli are thus given by

$$
\boldsymbol{S}^{t}=\boldsymbol{S}+\sum_{\alpha} \frac{1}{h^{\alpha}} \hat{\boldsymbol{\epsilon}}^{\alpha} \otimes \hat{\boldsymbol{\epsilon}}^{\alpha}
$$

The summation in (82) is taken only over those transformation systems that are active by satisfaction of (74). So far, no attention has been given to the requirement that the switching process saturates when any of the $v^{I}$ falls to zero. This requirement can be met by making the $h^{\alpha}$ functions of $v^{I}$ that grow large as $v^{I} \rightarrow 0$. Alternatively, incremental calculations can be carried out by testing for $v^{I}>0$ at each step and suppressing any transformation that violates this condition. Care must also be taken to arrange that, during cyclical loading, the accumulated hardening of each transformation system is reversed during the cycle to allow for the stable cyclic hysteresis observed in most ferroelectrics. Since the transformation systems that convert $I \rightarrow J$ and $J \rightarrow I$ are separate, this requires softening of inactive transformation systems when the corresponding $v^{I}$ move away from zero. Approximate incremental calculations have been achieved by setting the $h^{\alpha}$ values to be small, and neglecting changes in $G^{\alpha c}$ (Huber and Fleck (2004)).

Having established the tangent moduli for a single crystal or grain, the response of a polycrystal can be computed by treating the polycrystal as a composite of a number of grains, using the methods discussed in section 3. If the self-consistent method is used, iteration is required to find 
the stress and electric field in each grain as the tangent moduli are dependent on $\boldsymbol{\sigma}$ through (77); this method has been shown to be in good agreement with more sophisticated, but computationally expensive, finite element calculations by Haug et al. (2007). Also, Huber and Fleck (2004) have demonstrated that the model can be fitted to various ferroelectric materials, though the fitting process is not straightforward, since neither the microscopic moduli $\boldsymbol{S}^{I}$ nor the $G^{\alpha c}$ are readily available for most materials. The rate-independent formulation developed here can be replaced with a rate-dependent formulation by making the transformation rates $\dot{f}^{\alpha}$ depend directly on $G^{\alpha}$ without requiring a switching criterion to be satisfied. This method has the advantage of avoiding the need for hardening relationships, and providing a simple, forward calculation; the rate-dependent formulation has been used by several researchers (Huber and Fleck (2001); Kamlah et al. (2005); Haug et al. (2007); Pathak and McMeeking (2008)) for practical calculations.

\subsection{Example of crystal plasticity model}

To illustrate the crystal plasticity approach to modelling ferroelectrics, the response of a single crystal of polar tetragonal material is simulated here. The six crystal variants have spontaneous polarization states as given by (10) and the corresponding spontaneous strain states given by (11). In the initial state the volume fractions of the crystal variants are taken to be $v^{I}=1 / 6, I=1 \ldots 6$. For simplicity the variants are here modelled as having isotropic elasticity and dielectric permittivity given by

$$
\begin{aligned}
s_{i j k l}^{E} & =\frac{1+\nu}{2 Y}\left(\delta_{i k} \delta_{j l}+\delta_{j k} \delta_{i l}\right)-\frac{\nu}{Y} \delta_{i j} \delta_{k l} \\
\varepsilon_{i j}^{\sigma} & =\varepsilon \delta_{i j}
\end{aligned}
$$

where $Y$ is Young's modulus, $\nu$ is Poisson's ratio and $\varepsilon$ is the dielectric permittivity at constant stress. The piezoelectric tensor is expected to be strongly anisotropic and is given by

$$
\begin{aligned}
d_{k i j}^{I}=d_{33} p_{k}^{I} p_{i}^{I} p_{j}^{I}+d_{31}\left(p_{k}^{I} \delta_{i j}-p_{k}^{I} p_{i}^{I} p_{j}^{I}\right) \\
+d_{15}\left(\delta_{k i} p_{j}^{I}+\delta_{k j} p_{i}^{I}-2 p_{k}^{I} p_{i}^{I} p_{j}^{I}\right)
\end{aligned}
$$

where $\boldsymbol{p}^{\boldsymbol{I}}=\boldsymbol{P}^{\boldsymbol{I}} / P_{0}$ is the unit vector in the direction of the spontaneous polarization for each crystal variant; $d_{33}, d_{31}$ and $d_{15}$ are piezoelectric constants. With these definitions, $\Delta \boldsymbol{S}^{\alpha}$ becomes

$$
\Delta \boldsymbol{S}^{\alpha}=\boldsymbol{S}^{J}-\boldsymbol{S}^{I}=\left(\begin{array}{cc}
0 & d_{k i j}^{J}-d_{k i j}^{I} \\
d_{i k l}^{J}-d_{i k l}^{I} & 0
\end{array}\right)
$$


Table 2. Material data and model parameters for the tetragonal crystal plasticity model.

\begin{tabular}{lclc}
\hline$E(\mathrm{GPa})$ & 60 & $P_{0}\left(\mathrm{C} \mathrm{m}^{-2}\right)$ & 0.25 \\
$\nu$ & 0.3 & $\epsilon_{0}$ & $0.2 \%$ \\
$\varepsilon\left(\mathrm{nF} \mathrm{m}^{-1}\right)$ & 22.5 & $G^{\alpha c}\left(180^{\circ}\right)\left(\mathrm{MJ} \mathrm{m}^{-3}\right)$ & 0.5 \\
$d_{33}\left(\mathrm{pm} \mathrm{V}^{-1}\right)$ & 315 & $G^{\alpha c}\left(90^{\circ}\right)\left(\mathrm{MJ} \mathrm{m}^{-3}\right)$ & 0.25 \\
$d_{31}\left(\mathrm{pm} \mathrm{V}^{-1}\right)$ & -128 & $\mathrm{n}$ & 5 \\
$d_{15}\left(\mathrm{pm} \mathrm{V}^{-1}\right)$ & 482 & & \\
\hline
\end{tabular}

At each step in the simulation, (73) is used to compute the driving force for switching by each transformation system, but instead of finding the tangent moduli of (82), a rate-dependent formulation is used by setting (Huber and Fleck (2001); Pathak and McMeeking (2008))

$$
\dot{f}^{\alpha}=H^{\alpha}\left|\frac{G^{\alpha}}{G^{\alpha c}}\right|^{n-1} \frac{G^{\alpha}}{G^{\alpha c}}
$$

which, for values of $n \gg 1$ has the effect of suppressing switching when $G^{\alpha}<G^{\alpha c}$ and allowing switching to proceed rapidly whenever $G^{\alpha}>G^{\alpha c}$. To provide saturation of switching as $v^{I} \rightarrow 0$, Pathak and McMeeking (2008) introduced the hardening rule

$$
H^{\alpha}=1-\mathrm{e}^{-v^{I} / v^{0}}
$$

with $v^{0}=0.01$. Material parameters similar to those of Pathak and McMeeking (2008) have been used (see table 2), except for the use of isotropic elastic and dielectric moduli. Note that distinct values of $G^{\alpha c}$ are used for $90^{\circ}$ switching and $180^{\circ}$ switching. This has the effect that the critical value of electric field at which rapid ferroelectric switching occurs is about the same for the two distinct types of switching. In the model, the crystal is subjected to cyclic electric field loading of the form $E_{1}=2 E_{0} \sin (\omega t)$ with $\omega=2 \pi \mathrm{rad} \mathrm{s}^{-1}$ and $E_{0}=G^{\alpha c}\left(90^{\circ}\right) / P_{0}$. The stress is held at zero throughout. The $\dot{f}^{\alpha}$ are computed using (87) and integrated over small time steps to update the volume fractions. At each time step, the remanent quantities $\epsilon_{i j}^{r}$ and $P_{i}^{r}$ are updated using (66) and the material moduli are updated using (67), with only the piezoelectric moduli changing, as in (86).

Starting from the initial condition with all $v^{I}=1 / 6$ the electric field $E_{1}$ increases, causing switching to increase the volume fraction of the crystal variant with $P_{1}^{J}=P_{0}$ at the expense of the other five variants. Eventually this process saturates with $\boldsymbol{P}^{r}=P_{0}\left(\begin{array}{lll}1 & 0 & 0\end{array}\right)^{T}$. Later in the cycle, when $E_{1}<0$ the switching process is reversed. The volume fraction of the variant 

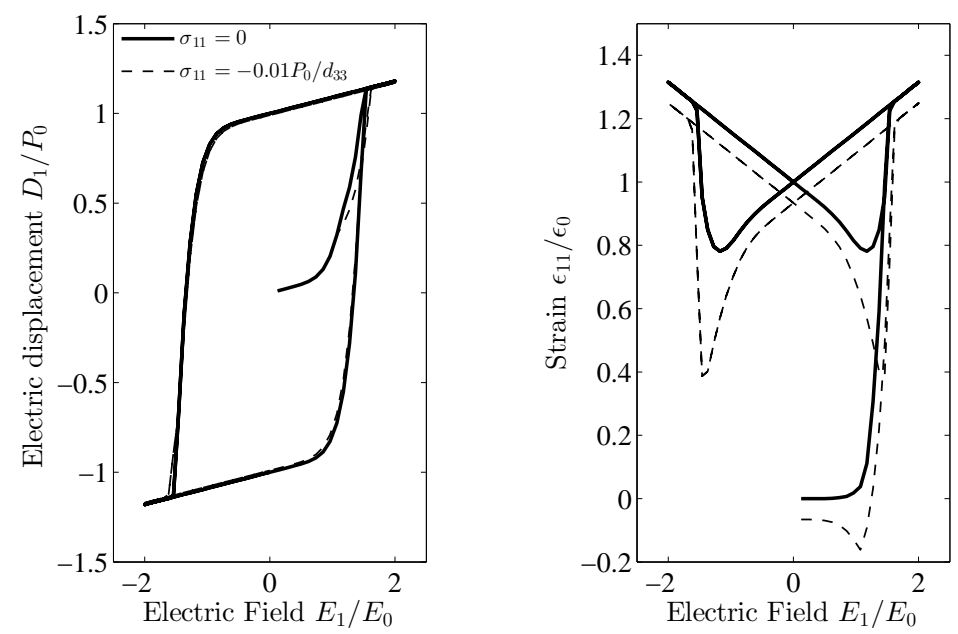

Figure 5. Electric displacement and strain hysteresis loops for a tetragonal single crystal, modelled by the crystal plasticity method, and showing the effect of a compressive stress.

with $P_{1}^{J}=-P_{0}$ increases, and similarly the volume fractions of variants with polarization states orthogonal to the applied field increase. However, as this happens, further switching transfers volume fraction from these orthogonal variants into the $P_{1}^{J}=-P_{0}$ variant, which eventually reaches a volume fraction of unity, so that $\boldsymbol{P}^{r}=-P_{0}\left(\begin{array}{lll}1 & 0 & 0\end{array}\right)^{T}$. As the electric field is cycled, the polarization state cycles between $\pm P_{0}\left(\begin{array}{lll}1 & 0 & 0\end{array}\right)^{T}$. Figure 5 shows the resulting hysteresis in electric displacement versus electric field, calculated using (62). Also shown is the variation of the strain component $\epsilon_{11}$ which follows the "butterfly" hysteresis pattern characteristic of ferroelectrics. This arises due to a combination of effects: the piezoelectric moduli of the crystal reverse during each half cycle of loading, giving rise to regions with positive and regions with negative slope $\partial \epsilon_{11} / \partial E_{1}$. The changes in volume fractions also affect the remanent strain, producing a large increase in strain during the first quarter cycle of loading and affecting the shape of the subsequent hysteresis.

Figure 5 also shows, in dashed lines, the effect on the hysteresis curves of a moderate compressive stress $\sigma_{11}=-0.01 P_{0} / d_{33}$, which is equivalent to about $8 \mathrm{MPa}$ compression. This stress is held constant throughout, while the electric field is again cycled. The compression has only a slight effect 
on the dielectric hysteresis curve, but the strain hysteresis is substantially changed. Of interest, the presence of compressive stress greatly increases the amplitude of strain variation during the stable cyclic hysteresis. This happens because the compressive stress stabilizes the four crystal variants with polarization orthogonal to the electrical loading axis. These crystal variants have negative $\epsilon_{11}^{I}$ components which causes the strain response to curve downwards during switching, and then turn sharply upwards as switching proceeds favouring the crystal variant whose spontaneous polarization is aligned with the electric field. A practical consequence is that the strain of piezoelectric actuators can be enhanced by holding them under moderate compression. This encourages an "extrinsic" contribution to the piezoelectric response, caused by the movement of domain walls. Piezoelectric actuators are commonly held under such a bias stress to improve their performance. Similar effects were observed in barium single titanate crystals by Burcsu et al. (2004).

The model described in this section can be incorporated into finite element calculations (Haug et al. (2007); Pathak and McMeeking (2008)) enabling simulation of the response of polycrystalline materials, and allowing complex boundary conditions to be handled. The crystal plasticity approach is also attractive in that the model contains an ample quantity of internal variables in the form of the volume fractions $v^{I}$, and these relate naturally to both the switching process and the saturation of switching. Furthermore, the $v^{I}$ and crystallographic orientations can be inferred from X-ray or neutron diffraction studies (Hall et al. (2005); Jones et al. (2005)) enabling comparison of models with experimental data at both the macroscopic and microscopic scales. However, as noted by Pathak and McMeeking (2008), there are deficiencies. The Reuss-type assumption of uniform stress and electric field at the grain level is unlikely to be accurate. Also, the model allows that any crystal variant can transform into any other. In practice, specific structures or patterns of domains form within ferroelectric crystals and these limit the available transformation systems. This factor motivates modelling at a finer scale to capture the effects of domain pattern.

\section{Models of Ferroelectric Domain Patterns}

In section 4.3 the observation was made that specific structures or patterns of domains may influence the behaviour of a ferroelectric crystal. Hence there arises the possibility of arranging the domains within a crystal in an advantageous way. For example, in a crystal with engineered domain configuration (Park and Shrout (1997); Liu and Lynch (2006); Wu et al. (2011)), enhanced piezoelectric properties are achieved by arranging 
a pattern of favourably oriented domains, or aligning a crystal such that favourable transformations happen when electric field is applied. There is also the opportunity to design new ferroelectrics with favourable properties by understanding the process by which domain patterns form and their consequences for the mechanism of ferroelectric switching - similar methods have been applied in the context of martensites (James and Hane (2000); Zhang and James (2009)). Finally, an improved representation of domain patterns could be incorporated into models of the type developed in section 4.2 for better accuracy.

Experimental evidence (Arlt (1990); Tsou et al. (2011)) makes clear that laminated patterns of domains are common and this observation can be interpreted as a consequence of energy minimization, similar to the formation of laminates in the theory of martensite (Ball and James (1987); Bhattacharya (1993)). The energy minimization approach has been used to predict domain patterns in ferroelectric crystals (Shu and Bhattacharya (2001)) and hence obtain the piezoelectric properties of poled crystals with engineered domain configurations ( $\mathrm{Li}$ and Liu (2004)). The key step in the energy minimization approach is to recognize (Shu and Bhattacharya (2001); Ball and James (1987)) that the overall energy is minimized when the strain and polarization state at almost every material point belongs to one of the set of minima of a multi-well free energy function. Consider a ferroelectric occupying volume $V$ which is part of the extended space (volume $\tilde{V}$ ) that contains free space outside $V$. Let the material be subjected to tractions $t_{i}$ and surface charges of density $q$ over a portion of its surface. Then write the energy to be minimized as:

$$
\int_{V} \Psi_{\text {bulk }}(\boldsymbol{\epsilon})+\Psi_{\text {grad }} d V-\int_{S} t_{i} u_{i}+\phi q d S+\int_{\tilde{V}} \frac{1}{2} \varepsilon_{0} \phi_{, i} \phi_{, i} d \tilde{V}
$$

The physical meaning of the terms in (89) is as follows: $\Psi_{\text {bulk }}$ is the bulk energy, depending on the strain and electric displacement at each point in the region $V$ occupied by the ferroelectric. This term has the multi-well structure discussed in section 2 . In the ferroelectric phase, $\Psi_{\text {bulk }}$ can without loss of generality be defined such that $\Psi_{\text {bulk }} \geq 0$ with $\Psi_{\text {bulk }}(\epsilon)=0$ for any $\boldsymbol{\epsilon}=\boldsymbol{\epsilon}^{I}, I \in 1 \ldots N$, corresponding to the set of spontaneous strain and polarization states of the crystal variants. The term $\Psi_{\text {grad }}$ is the additional energy due to local gradients in polarization. Typically, this term is assumed to depend only on the gradient $P_{i, j}$ of the conventional polarization, which is that part of the electric displacement due to the presence of the material in free space; that is,

$$
D_{i}=P_{i}+\varepsilon_{0} E_{i}
$$


where $\varepsilon_{0}$ is the permittivity of free space, $8.854 \times 10^{-12} \mathrm{~F} \mathrm{~m}^{-1}$. The meaning of polarization in this context is different from the remanent polarization discussed in sections 2-4: $P_{i}$ includes both the spontaneous polarization $P_{i}^{I}$ of the domain and that part of the reversible electric displacement resulting from the dielectric properties of the material. For example in a linear piezoelectric, using (9)

$$
P_{i}=e_{i k l} \epsilon_{k l}+\left(\varepsilon_{i k}^{\epsilon}-\varepsilon_{0} \delta_{i k}\right) E_{k}+P_{i}^{I}
$$

Including the gradient energy is physically reasonable on the grounds that domain walls, where $P_{i, j}$ is non-zero, contribute to the overall energy of the system. But the domain walls have finite thickness, introducing a length scale that is associated with $\Psi_{\text {grad }}$. Continuing with the terms in (89), the surface integral contains the potential energy of external loads in the form of tractions $t_{i}$ displaced by $u_{i}$ and surface free charge density $q$ at voltage $\phi$, applied to the material. The final term includes the stored electrostatic energy of free space. This term should be modified if there is any material other than free space surrounding the ferroelectric in region $V$. However, changes in this final term due to loading on $V$ or rearrangement of the domains in $V$ are typically much smaller than the resulting changes in the bulk energy term and so the energy of free space is often (but not always see Dayal and Bhattacharya (2007)) neglected.

Given a specific form for $\Psi_{\text {bulk }}$ and $\Psi_{\text {grad }}$, (89) can be minimized to yield patterns of polarization throughout a ferroelectric body, consistent with applied boundary conditions. The use of gradient methods to relax the energy towards the minimum results in Ginzburg-Landau type phasefield models which have been widely used to study ferroelectric domains. For periodic problems, Fourier spectral methods are expedient (Wang et al. (2013)), while real space solutions can be found using finite difference, finite element and boundary element methods (Wang et al. (2009); Su and Landis (2007); Dayal and Bhattacharya (2007)). Phase-field models are not described in further detail in this chapter. However, it should be noted that, in numerical models, it is necessary to discretize space at a scale finer than the domain wall width. Typically, a minimum of about 3 elements across a domain wall is needed (Völker and Kamlah (2012)), resulting in a mesh spacing of $<10^{-9} \mathrm{~m}$. The resulting models are computationally expensive, but have the advantage of allowing a great diversity of problems to be studied. A further point to note is that the phase-field models will typically identify a unique minimum, or in time-dependent formulations, a unique path of pattern evolution. This is of course desirable on physical grounds. However, due to the multi-well structure of the bulk energy, multiple local minima may exist and repeat runs of these models with slightly different 
starting conditions can produce qualitatively different results.

\subsection{Theory of compatibility}

The remainder of this section is devoted to the constrained theory of ferroelectric domains. The constrained theory arises from neglecting the term $\Psi_{\text {grad }}$ in (89), recognizing that the domain walls in ferroelectrics are of thickness only a few times the lattice parameter and the domain wall energy makes no significant contribution when the size of region $V$ is much greater than the domain wall thickness. Then, considering the case without external loads, the minimisation problem reduces to finding an arrangement of $\epsilon$ throughout $V$ that minimizes $\int_{V} \Psi_{\text {bulk }} d V$. This can be achieved by giving each point (except those within domain walls, which are taken to constitute a negligible volume) a strain and polarization belonging to one of the set of $\epsilon^{I}$ of the symmetry related variants. Setting

$$
\boldsymbol{\epsilon}=\boldsymbol{\epsilon}^{I}, \quad I \in 1,2, \ldots, N
$$

gives immediately $\int_{V} \Psi_{\text {bulk }} d V=0$. But this also produces particular requirements on the arrangement of domains and of any domain walls present. Consider a domain wall with normal $\boldsymbol{n}$ separating domains in states $\boldsymbol{\epsilon}^{1}$ and $\boldsymbol{\epsilon}^{2}$. Then, from (20):

$$
\left(P_{i}^{2}-P_{i}^{1}\right) n_{i}=0
$$

Similarly, considering the jump in strain and requiring continuity of displacement, (23) results in the Hadamard jump condition (Shu and Bhattacharya (2001)) which can be written, in the context of small strains, as

$$
\epsilon_{i j}^{2}-\epsilon_{i j}^{1}=n_{i} a_{j}+n_{j} a_{i}
$$

for some vector $\boldsymbol{a}$. Now, since $\boldsymbol{\epsilon}^{1}$ and $\boldsymbol{\epsilon}^{2}$ must be drawn from the set of spontaneous states of the domains, it is possible that, for a given pair of domains, the compatibility equations (93) and (94) have no solutions. There can be at most two non-trivial solutions for $\boldsymbol{n}$ (Ball and James (1987)). However, there are also degenerate cases. For example it is common in ferroelectrics to have two variants with identical spontaneous strain states but opposite polarization. Then (94) is satisfied by any $\boldsymbol{n}$ with $\boldsymbol{a}=0$ and (93) only restricts $\boldsymbol{n}$ to lie in the plane orthogonal to $\boldsymbol{P}^{2}-\boldsymbol{P}^{1}$, producing a continuous set of domain wall normals. This results in $180^{\circ}$ walls without a unique orientation, and gives rise to wavy patterns commonly seen in ferroelectric microstructure. More generally, the left hand side of (94) is symmetric so that orthogonal eigenvectors $\boldsymbol{e}_{1}, \boldsymbol{e}_{2}$ and $\boldsymbol{e}_{3}$ can be identified, with corresponding eigenvalues $\lambda_{1}, \lambda_{2}$ and $\lambda_{3}$. For solutions of (94) to exist, 
there is at least one eigenvalue that is zero: let $\lambda_{3}=0$. Additionally, because the spontaneous strains are those of symmetry related variants any one of which can be derived from any other by a pure rotation, the eigenvalues sum to zero, so $\lambda_{1}=-\lambda_{2}$. Then solutions of (94) give the interface normal as

$$
\boldsymbol{n}=\frac{\boldsymbol{e}_{1} \pm \boldsymbol{e}_{2}}{\sqrt{2}}
$$

and the resulting $\boldsymbol{n}$ can be tested for electrical compatibility using (93). Ball and James (1987) and Shu and Bhattacharya (2001) have give a fuller discussion of the compatibility equations in the context of finite deformation gradients. Note that if a unique $\boldsymbol{n}$ results as the solution to (93) and (94), then the pair of domains can form only one orientation of domain wall consistent with energy minimization. This typically results in laminated microstructure with the layers alternating between two crystal variants.

What states of macroscopic remanent strain and polarization can a crystal achieve while satisfying the requirements of compatibility? Suppose that a region of a ferroelectric crystal in volume $V$ is subjected to surface displacements and charges $u_{i}, q$, relative to a reference state with $\epsilon=0$, such that $u_{i}=\tilde{\epsilon}_{i j} x_{j}$ and $-q=\tilde{D}_{i} n_{i}$ for some $\tilde{\boldsymbol{\epsilon}}=\left(\tilde{\epsilon}_{i j}, \tilde{D}_{i}\right)$, where $n_{i}$ is the surface normal. Let the resulting pattern of domains form on a scale much finer than that of the region $V$, and homogeneous across the region. Then a compatible arrangement of domains with average strain and polarization given by $\overline{\boldsymbol{\epsilon}}=\tilde{\boldsymbol{\epsilon}}$ that can be periodically repeated across the region achieves a minimum energy state in $V$ with stress and electric field everywhere equal to zero. Care must be taken in applying the small strain formulation of the compatibility equations (93) and (94) to this problem. Specifically, (94) neglects the small rotations in crystal lattice orientation that may occur at domain walls. This point will briefly be discussed later on. First, however, consider specific arrangements of domains in the form of a hierarchical laminate that satisfies (93) and (94) in an average sense at every internal interface.

\subsection{Average compatibility}

A hierarchical arrangement of laminations can guarantee satisfying compatibility conditions and matching a prescribed macroscopic state of remanent strain and polarization $\boldsymbol{\epsilon}^{r}$ by construction. First note that within the constrained theory, each material point has strain and electric displacement matching one of the $N$ states $\boldsymbol{\epsilon}^{I}, I=1 \ldots N$. Implicitly, each domain has zero stress and electric field, so that, in the absence of external loading, $\boldsymbol{\epsilon}^{r}=\overline{\boldsymbol{\epsilon}}=\tilde{\boldsymbol{\epsilon}}=\overline{\boldsymbol{\epsilon}^{I}}$. That is, the strain and electric displacement is entirely due to spontaneous states of the crystal variants. 


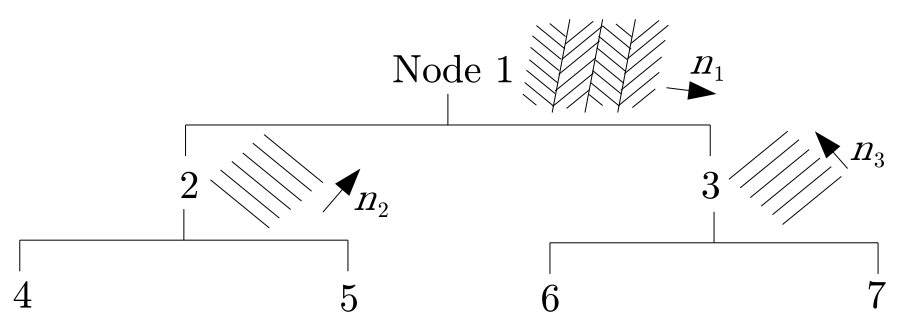

Figure 6. Tree diagram corresponding to a rank-2 laminate, showing interface normals and pattern of laminations.

To form a hierarchical laminate, a pair of crystal variants are first laminated with domain walls normal to $\boldsymbol{n}$, derived by solving (93) and (94). The resulting laminate is formed with sufficiently fine layers to be treated as a homogenized medium with effective $\boldsymbol{\epsilon}$ that can be computed as a volume average over the constituent layers. The result is a rank-1 laminate. Higher rank laminates can be formed by laminating together layers of two materials, each of which is formed as a lower rank lamination - a rank $R$ lamination being formed from fine layers comprising two rank $R-1$ laminations. The resulting hierarchy of laminates can be visualized using a binary tree diagram (see figure 6) in which the root node (node number 1) represents the macroscopic material, comprising laminations of materials represented by nodes 2 and 3 and so forth. A rank $R$ lamination has a corresponding tree diagram with $R+1$ layers and $2^{R+1}-1$ nodes.

The nodes in layer $R+1$ each represent material comprising a single variant drawn from the set of $N$ crystal variants. Numbering the nodes in the tree diagram from the top down and from left to right as shown in figure 6 , each node $i$ in layers $1 \ldots R$ has an associated interface normal, $\boldsymbol{n}_{i}$ defining the orientation of its constituent layers. Each node also has an associated volume fraction $f_{i}$ such that $f_{1}=1=f_{2}+f_{3}$ and so forth:

$$
f_{i}=f_{2 i}+f_{2 i+1}, \quad i=1 \ldots 2^{R}-1
$$

and since each lamination satisfies the compatibility conditions,

$$
f_{i} \boldsymbol{\epsilon}_{i}^{r}=f_{2 i} \epsilon_{2 i}^{r}+f_{2 i+1} \epsilon_{2 i+1}^{r}, \quad i=1 \ldots 2^{R}-1
$$

As usual, $\boldsymbol{\epsilon}^{r}$ is used as compact notation representing both remanent strain and polarization. Each of the $N$ variants has some volume fraction $f^{I} \geq 0$, $I=1 \ldots N$ where the superscript $I$ distinguishes volume fractions of the crystal variants from the volume fractions $f_{i}$ associated with the nodes. 
Again, the compatibility conditions enforce

$$
\boldsymbol{\epsilon}^{r}=\sum_{1}^{N} f^{I} \boldsymbol{\epsilon}^{I}
$$

with

$$
\sum_{1}^{N} f^{I}=1
$$

If the macroscopic remanent strain and polarization are specified, (98) and (99) give rise to a system of linear equations for the $N$ volume fractions $f^{I}$. The strain component of (98) gives 6 linear equations; the polarization part gives 3 equations and (99) provides one further equation. Hence there are 10 equations for the $N$ unknowns, with coefficients dependent on the spontaneous strain and polarization states of the crystal. It is immediately evident that the crystal parameters can result in (98) and (99) being invertible, singular or over-determined. Particular cases will be examined in detail in section 5.4.

Suppose now that the $f^{I}$ are known. Following Bhattacharya (1993) and Li and Liu (2004) it is possible to construct a compatible lamination with these overall variant volume fractions as follows. Rewrite (98) as

$\boldsymbol{\epsilon}^{r}=\mu_{1} \epsilon^{1}+\mu_{2}\left(1-\mu_{1}\right) \epsilon^{2}+\cdots+\mu_{N-1} \prod_{m=1}^{N-2}\left(1-\mu_{m}\right) \epsilon^{N-1}+\prod_{m=1}^{N-1}\left(1-\mu_{m}\right) \epsilon^{N}$

where the coefficients $\mu_{m}$ are given by

$$
\begin{aligned}
& \mu_{m}=0 \text { if } \sum_{I=1}^{m-1} f^{I}=1, \\
& \mu_{m}=\frac{f^{m}}{1-\sum_{I=1}^{m-1} f^{I}} \text { otherwise }
\end{aligned}
$$

The volume fractions $f_{i}$ of the material represented by each node in the lowest level of the rank-R binary tree diagram can then be reconstructed using (Tsou and Huber (2010)):

$$
f_{i}=\prod_{k=1}^{R}(1-2 b) \mu_{k}+b, \quad i=2^{R} \ldots 2^{R+1}-1
$$




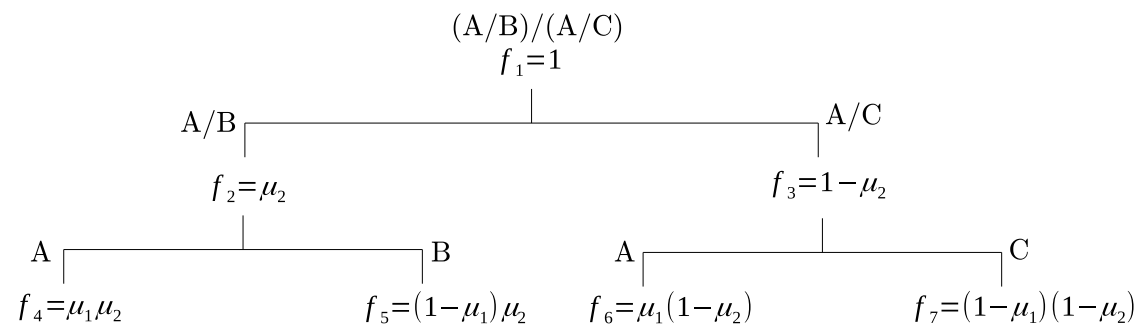

Figure 7. A rank-2 tree diagram showing the construction of volume fractions $f_{i}$ from $\mu_{k}$.

with

$$
b=\left\lfloor\frac{i-2^{R}}{2^{k-1}}\right\rfloor-2\left\lfloor\frac{i-2^{R}}{2^{k}}\right\rfloor
$$

where $\lfloor x\rfloor$ represents the floor function that gives the greatest integer less than or equal to $x$. Volume fractions associated with node numbers $i<2^{R}$ are recovered by successive applications of (96). The binary digit $b$ defined in (103) takes on the values zero or unity to indicate the branch of the tree diagram in which node $i$ resides. If node $i$ is reached via the left hand branch at level $k-1$ then $b=0$; otherwise $b=1$. The resulting pattern of volume fractions in a rank-2 tree diagram is shown in figure 7 , along with labels $(\mathrm{A}, \mathrm{B}, \mathrm{C})$ to indicate the crystal variants represented at each node. Provided that the individual crystal variants are pairwise compatible, that is, there exists a domain wall orientation satisfying (93) and (94) for any pairing of crystal variants, then the arrangement of volume fractions specified by (100-103) guarantees that a fully compatible hierarchical laminate can form with average strain and polarization state $\boldsymbol{\epsilon}^{r}$. The way this arrangement produces compatibility is evident from figure 7. Variant A is repeated in laminations $\mathrm{A} / \mathrm{B}$ and $\mathrm{A} / \mathrm{C}$ with identical volume fractions. Since A can form a notional interface with itself in any orientation, the only requirement to form a lamination between $\mathrm{A} / \mathrm{B}$ and $\mathrm{A} / \mathrm{C}$ is that $\mathrm{B}$ can form an interface with $\mathrm{C}$; this requirement is met by pairwise compatibility. In terms of the average strains and polarizations,

$$
\begin{aligned}
& \boldsymbol{\epsilon}_{2}=\mu_{1} \boldsymbol{\epsilon}_{4}+\left(1-\mu_{1}\right) \boldsymbol{\epsilon}_{5}=\mu_{1} \boldsymbol{\epsilon}^{A}+\left(1-\mu_{1}\right) \boldsymbol{\epsilon}^{B} \\
& \boldsymbol{\epsilon}_{3}=\mu_{1} \boldsymbol{\epsilon}_{6}+\left(1-\mu_{1}\right) \boldsymbol{\epsilon}_{7}=\mu_{1} \boldsymbol{\epsilon}^{A}+\left(1-\mu_{1}\right) \boldsymbol{\epsilon}^{C}
\end{aligned}
$$

where superscripts on $\epsilon$ identify particular crystal variants, but subscripts identify node numbers in the binary tree. Now, from pairwise compatibility

$$
\epsilon_{i j}^{B}-\epsilon_{i j}^{C}=n_{i}^{B C} a_{j}+n_{j}^{B C} a_{i}
$$


and

$$
\left(P_{i}^{B}-P_{i}^{C}\right) n_{i}^{B C}=0
$$

From which it follows that

$$
\left(\boldsymbol{\epsilon}_{2}-\boldsymbol{\epsilon}_{3}\right)_{i j}=n_{i}^{B C} a_{j}^{\prime}+n_{j}^{B C} a_{i}^{\prime}
$$

with $a_{i}^{\prime}=\left(1-\mu_{1}\right) a_{i}$, and

$$
\left(\boldsymbol{P}_{2}-\boldsymbol{P}_{3}\right)_{i} n_{i}^{B C}=0
$$

Similar compatible interfaces are guaranteed throughout the laminate. Since the $\mu_{m}$ in (100) can be chosen arbitrarily in the range $0 \leq \mu \leq 1$ and a compatible laminate can still be found, equivalently the volume fractions $f^{I}$ can be arbitrarily chosen. It follows that the states of remanent strain and polarization $\boldsymbol{\epsilon}^{r}$ accessible by a zero energy compatible laminate include all states within the convex hull of the set of $\boldsymbol{\epsilon}^{I}$, the spontaneous states of the individual crystal variants, given by

$$
\boldsymbol{\epsilon}^{r}=\sum_{i=1}^{N} f^{I} \boldsymbol{\epsilon}^{I}, \quad f^{I} \geq 0, \quad \sum_{i=1}^{N} f^{I}=1
$$

Compatibility is achieved in an average sense: The homogenized materials represented by the nodes can form an interface satisfying electrical and mechanical compatibility conditions, but the fine scale domain walls formed at the interface between two laminates do not necessarily satisfy compatibility conditions. The resulting pattern is an energy minimizer provided that there is a separation of length scales between successive laminations, allowing each lamination to be treated as a homogenized medium. This separation of length scales is consistent with observed microstructure (Liu and Lynch (2006); Arlt and Sasko (1980)) where layers of very fine domains are seen to form.

Note, however, that the resulting pattern of crystal variants requires a lamination of rank $N-1$ to accommodate $N$ distinct variants. It is rare in practice to see such multiple levels of length scale separation in ferroelectric crystals, and this motivates consideration of other ways that energy minimizing domain patterns can form, such as the formation of laminates without separation of length scales, but with compatibility satisfied exactly at every domain wall.

\subsection{Exact compatibility}

Next consider the necessary conditions for the formation of a laminate in which the compatibility conditions are satisfied exactly at every domain 

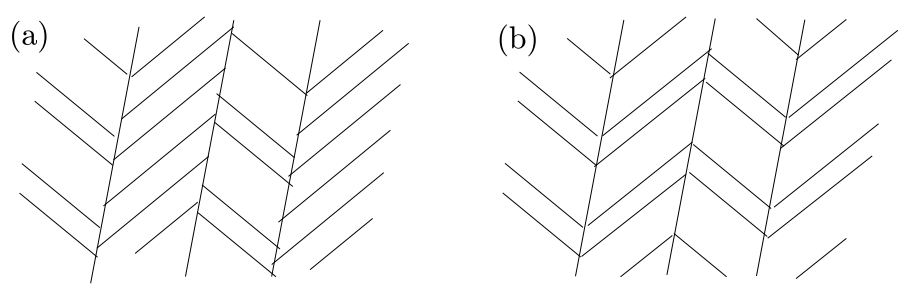

Figure 8. Rank-2 laminations with (a) unmatched volume fractions and (b) matched volume fractions according to equation (111).

wall. Tsou and Huber (2010) identified a set of sufficient conditions which guarantee such exact compatibility: First, wherever two laminations meet to form a higher-rank lamination, there should be a matching of volume fractions across the higher-rank interface. This avoids the type of mismatch indicated in figure 8a which, though it does not prevent the formation of exactly compatible interfaces, places extremely restrictive conditions upon their formation. With matched volume fractions, fewer pairings of crystal variants are formed. By reference to the binary tree diagram of rank- $R$, this first condition can be written as (Tsou and Huber (2010)):

$$
\frac{f_{2^{R-r}}}{f_{2^{R-r}+1}}=\frac{f_{2^{R-r}+2 n-2}}{f_{2^{R-r}+2 n-1}}, \quad n=2, \ldots, 2^{R-r-1}, \quad r=0 \ldots R-2
$$

This imposes a requirement upon all pairs of materials that are laminated together in a given level $r$ of the binary tree to share a common volume fraction ratio, allowing matched patterns such as figure $8 \mathrm{~b}$.

A second condition identified by Tsou and Huber (2010) is that pairs of domain walls in the two laminations that are joined to form a higher rank lamination intersect the common interface on the same line. This restricts the domain walls to have a common orientation when projected into the plane of that interface across which the higher rank lamination is formed. The condition forces the domain patterns on either side of an interface to match not only in spacing, as specified by (111) but also in orientation. The requirement can be written as a coplanarity condition on the interface normals associated with particular nodes in the binary tree:

$$
\boldsymbol{n}_{i} \cdot\left(\boldsymbol{n}_{p} \times \boldsymbol{n}_{q}\right)=0
$$

where $i, p$ and $q$ are node numbers selected as follows:

$$
p=2^{j+1} i+k, \quad q=p+2^{j},
$$


with $i, j$ and $k$ in the ranges

$$
i=1, \ldots, 2^{R-1}-1, \quad j=0, \ldots, r-2, \quad k=0, \ldots, 2^{j}-1
$$

and tree level $r=R-\left\lfloor\log _{2} i\right\rfloor$. The pattern of nodes picked out by (112114) can be illustrated by application to a rank-2 laminate. With $R=2$, the range of values specified in (114) becomes simply $i=1, j=0, k=0$. Then the node numbers $i, p, q$ for use in (112) are just $i=1, p=2, q=3$, requiring that $\boldsymbol{n}_{1}, \boldsymbol{n}_{2}$, and $\boldsymbol{n}_{3}$ are coplanar. When $R=3,(112-114)$ produce five coplanarity equations, with node numbers $i, p, q$ equal to

$$
\begin{array}{lll}
1, & 2, & 3 ; \\
1, & 4, & 6 ; \\
1, & 5, & 7 ; \\
2, & 4, & 5 ; \\
3, & 6, & 7
\end{array}
$$

Higher rank laminations lead to greater numbers of coplanarity equations and hence reduce the chance of finding an exactly compatible arrangement.

The final exact-compatibility condition for a rank- $\mathrm{R}$ lamination is that each domain walls satisfies (93) and (94), which brings in the spontaneous strain and polarization states of the individual domains. Once again, the set of nodes over which the compatibility equations apply must be specified. Write the compatibility equations as:

$$
\left(\boldsymbol{P}_{p}-\boldsymbol{P}_{q}\right) \cdot \boldsymbol{n}_{i}=0
$$

and

$$
\boldsymbol{\epsilon}_{p}-\boldsymbol{\epsilon}_{q}=\boldsymbol{n}_{i} \boldsymbol{a}_{i}+\boldsymbol{a}_{i} \boldsymbol{n}_{i}
$$

for nodes $i, p$ and $q$. The nodes $p, q$ are always drawn from the pure crystal variants in the lowest level of the tree diagram - nodes $2^{R}, \ldots, 2^{R+1}-1$. Meanwhile, the domain wall normal $\boldsymbol{n}_{i}$ is always associated with a higher level node $i<2^{R}$. The set of domain walls formed in the rank-R lamination is specified by:

$$
i=1, \ldots, 2^{R}-1, \quad p=2^{r} i+k, \quad q=p+2^{r-1}
$$

with

$$
k=0, \ldots, 2^{r-1}-1, \quad r=R-\left\lfloor\log _{2} i\right\rfloor
$$

In the case of a rank-2 laminate, (117) and (118) produce the node numbers $i, p, q$ equal to

$\begin{array}{lll}1, & 4, & 6 ; \\ 1, & 5, & 7 \\ 2, & 4, & 5 \\ 3, & 6, & 7\end{array}$


while in a rank-R laminate, $R \cdot 2^{R-1}$ sets of node numbers are produced. These conditions become highly restrictive in laminates of rank $R>2$ and are unlikely to be satisfied by sets of crystal variants with arbitrary spontaneous states $\boldsymbol{\epsilon}^{I}$. However, the crystal variants of typical ferroelectrics are both symmetric and symmetry related, with the result that exactly compatible laminates can often form.

It should be emphasized that the conditions specified by (111-118) are sufficient conditions for an exactly compatible laminate pattern, but are not necessary conditions for exactly compatible patterns of domains: other patterns such as crossing domains (Shu and Bhattacharya (2001)) can form and the microstructure is of course not restricted to form only binary laminates. However, Tsou et al. (2011) have shown that this formulation generates many of the commonly observed patterns of domains, and can give insight into observed domain patterns.

Returning to the problem of accessible states of macroscopic remanent strain and polarization, it was noted in section 5.2 that any state $\boldsymbol{\epsilon}^{r}$ that can be made up as the volume average over a set of pairwise compatible variants with states $\epsilon^{I}$ admits an averagely compatible laminate to form. However, the same is not true for exactly compatible laminates and the set of accessible states is in this case not known.

The theory of compatibility described in sections 5.1-5.3 uses the linear approximation in which deformation gradients are infinitesimal and deformation is represented by a strain tensor that is the symmetrized deformation gradient: the skew-symmetric part of the deformation gradient, representing rotation, is neglected. In typical ferroelectrics such rotations are small and the predictions of the linear theory are similar to those of the nonlinear theory. However, as noted by Bhattacharya (1993), significant errors can occur in the linear theory where there are multiple rotations. This can arise at the junction of domains. To see this, consider a path crossing a domain wall at which there is a net rotation of crystal lattice planes. Now a closed path around the junction of domains can cross several such walls. If the total lattice rotation is non-zero, then the formation of the junction from a continuous region of crystal results in a disclination and the loss of stress/electric field-free perfect compatibility. To account for the finite rotations, the use of the non-linear theory (Shu and Bhattacharya (2001)) is expedient. Alternatively, using the linear theory, Tsou and Huber (2010) computed the net rotation of lattice planes at each domain wall and used this to check for the existence of disclinations. 


\subsection{Examples of compatible laminates}

To illustrate the use of the theory of compatibility, consider ferroelectric crystals in the polar tetragonal system, which includes $\mathrm{BaTiO}_{3}$ and many other ferroelectrics. Here the crystal has six variants with spontaneous polarization states expressed in co-ordinates aligned with the crystallographic axes as follows:

$$
\begin{array}{ll}
\boldsymbol{P}^{1}=P_{0}\left(\begin{array}{l}
1 \\
0 \\
0
\end{array}\right) ; & \boldsymbol{P}^{2}=P_{0}\left(\begin{array}{c}
-1 \\
0 \\
0
\end{array}\right) ; \\
\boldsymbol{P}^{3}=P_{0}\left(\begin{array}{c}
0 \\
1 \\
0
\end{array}\right) ; & \boldsymbol{P}^{4}=P_{0}\left(\begin{array}{c}
0 \\
-1 \\
0
\end{array}\right) ; \\
\boldsymbol{P}^{5}=P_{0}\left(\begin{array}{c}
0 \\
0 \\
1
\end{array}\right) ; & \boldsymbol{P}^{6}=P_{0}\left(\begin{array}{c}
0 \\
0 \\
-1
\end{array}\right)
\end{array}
$$

The corresponding spontaneous strain states are

$$
\begin{aligned}
& \boldsymbol{\epsilon}^{1}=\boldsymbol{\epsilon}^{2}=\epsilon_{0}\left(\begin{array}{ccc}
1 & 0 & 0 \\
0 & -\frac{1}{2} & 0 \\
0 & 0 & -\frac{1}{2}
\end{array}\right) ; \\
& \boldsymbol{\epsilon}^{3}=\boldsymbol{\epsilon}^{4}=\epsilon_{0}\left(\begin{array}{ccc}
-\frac{1}{2} & 0 & 0 \\
0 & 1 & 0 \\
0 & 0 & -\frac{1}{2}
\end{array}\right) ; \\
& \boldsymbol{\epsilon}^{5}=\boldsymbol{\epsilon}^{6}=\epsilon_{0}\left(\begin{array}{ccc}
-\frac{1}{2} & 0 & 0 \\
0 & -\frac{1}{2} & 0 \\
0 & 0 & 1
\end{array}\right)
\end{aligned}
$$

Application of (93) and (94) to the polar tetragonal system shows that $180^{\circ}$ domain walls can form between variants 1 and 2 , variants 3 and 4 or variants 5 and 6 while $90^{\circ}$ domain walls form between any other pairs of variants. Application of (98) shows immediately that all $\epsilon_{i j}^{r}, i \neq j$ must be zero: the crystal cannot achieve remanent strain states that include shear components in co-ordinates aligned with the crystallographic axes. The remaining, non-trivial equations derived from (98) and (99) can be written 
as:

$$
\left[\begin{array}{c}
\epsilon_{11}^{r} / \epsilon_{0} \\
\epsilon_{22}^{r} / \epsilon_{0} \\
\epsilon_{33}^{r} / \epsilon_{0} \\
P_{1}^{r} / P_{0} \\
P_{2}^{r} / P_{0} \\
P_{3}^{r} / P_{0} \\
1
\end{array}\right]=\left[\begin{array}{cccccc}
1 & 1 & -\frac{1}{2} & -\frac{1}{2} & -\frac{1}{2} & -\frac{1}{2} \\
-\frac{1}{2} & -\frac{1}{2} & 1 & 1 & -\frac{1}{2} & -\frac{1}{2} \\
-\frac{1}{2} & -\frac{1}{2} & -\frac{1}{2} & -\frac{1}{2} & 1 & 1 \\
1 & -1 & 0 & 0 & 0 & 0 \\
0 & 0 & 1 & -1 & 0 & 0 \\
0 & 0 & 0 & 0 & 1 & -1 \\
1 & 1 & 1 & 1 & 1 & 1
\end{array}\right]\left[\begin{array}{l}
f^{1} \\
f^{2} \\
f^{3} \\
f^{4} \\
f^{5} \\
f^{6}
\end{array}\right]
$$

This has the form $\boldsymbol{e}=\boldsymbol{M} \boldsymbol{f}$ where $\boldsymbol{M}$ is a $7 \times 6$ matrix of rank 6 . Given $\boldsymbol{e}$, this yields a unique solution for the six volume fractions in $\boldsymbol{f}$ provided that the remanent strain and polarization state specified in $\boldsymbol{e}$ is within the convex hull of the set of spontaneous states. This result is special to the polar tetragonal system: the macroscopic $\epsilon^{r}$ prescribes the volume fractions $f^{I}$, but the arrangement of domains is non-unique. Consider, for example, a fully "unpoled" state, in which

$$
\epsilon^{r}=0
$$

so that

$$
\boldsymbol{e}=\left[\begin{array}{lllllll}
0 & 0 & 0 & 0 & 0 & 0 & 1
\end{array}\right]^{T}
$$

For this state, (121) gives the volume fractions

$$
\boldsymbol{f}=\left[\begin{array}{llllll}
\frac{1}{6} & \frac{1}{6} & \frac{1}{6} & \frac{1}{6} & \frac{1}{6} & \frac{1}{6}
\end{array}\right]^{T}
$$

Now the construction of Li and Liu (2004) and Bhattacharya (1993) gives the $\mu$ values for an averagely compatible laminate with this state of macroscopic $\boldsymbol{\epsilon}^{r}$. Using (101)

$$
\mu_{1}=\frac{1}{6} ; \quad \mu_{2}=\frac{1}{5} ; \quad \mu_{3}=\frac{1}{4} ; \quad \mu_{4}=\frac{1}{3} ; \quad \mu_{5}=\frac{1}{2} ;
$$

An averagely compatible laminate of rank-5 can match the "unpoled" state. The resulting laminate is, however, highly complicated, with the requirement for separation of length scales over multiple levels of hierarchical lamination. Considering exactly compatible laminates that may match the same $\boldsymbol{\epsilon}^{r}$, Tsou and Huber (2010) showed that a rank-3 exactly compatible laminate can form, see figure 9 . The rank-3 solution is not unique and many alternate arrangements are possible. The arrangement shown in figure 9 can be represented by a binary tree diagram with the crystal variants in the 


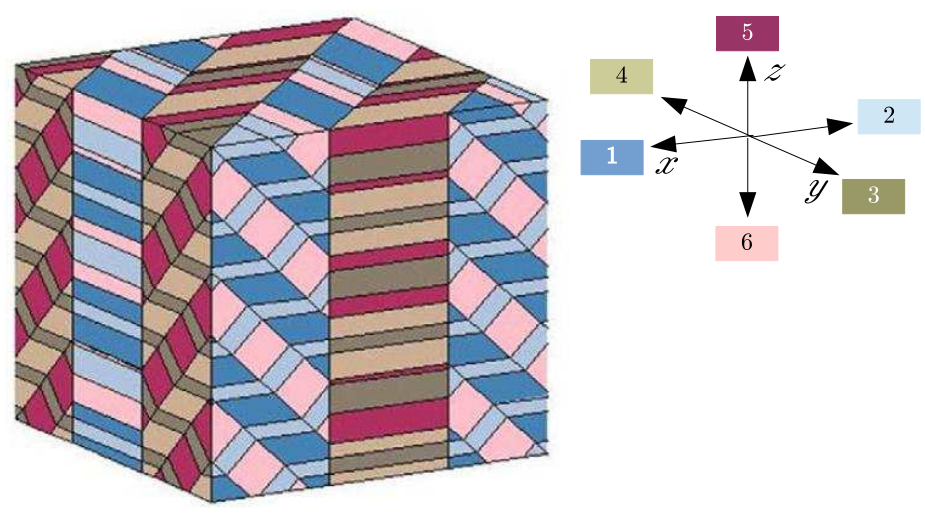

Figure 9. A rank-3 laminate in the polar tetragonal crystal system that achieves $\boldsymbol{\epsilon}^{r}=0$. Spontaneous polarization directions of the individual domains are indicated using the colour coding.

lowest level of the tree having numbers $1,2,6,2,4,3,5,3$ reading across the tree diagram from left to right. Use the notation " $I J$ " to represent a rank-1 lamination of variants $I$ and $J$, " $I J K L$ " to represent a rank-2 lamination comprising alternating layers of " $I J$ " and " $K L$ ", and so forth. Then, figure 9 shows the laminate"12624353". Note, however, that this numbering specifies only the arrangement of the crystal variants, not the orientation of domain walls or the volume fractions. In figure $9, f^{I}=1 / 6$ for each variant. The corresponding nodal volume fractions in the lowest level of the tree diagram are $f_{8}=1 / 6, f_{9}=1 / 12, f_{10}=1 / 6, f_{11}=1 / 12$, $f_{12}=1 / 6, f_{13}=1 / 12, f_{14}=1 / 6, f_{15}=1 / 12$.

It is of interest to study the variety of exactly compatible laminates that can form within a given crystal system. From consideration of the binary tree diagram, each node in the lowest level of the tree could represent any of the individual crystal variants. A rank-R binary tree has $2^{R}$ nodes in its lowest level, so in a crystal system with $N$ variants the lowest level of the tree diagram has $N^{2^{R}}$ possible arrangements. However, many of these may represent identical laminates, differing only by rotations or reflections. Furthermore, many of the arrangements may not satisfy the conditions for compatibility given in section 5.3.

Using the 6 variants of the polar tetragonal system defined by (119) and (120) as an example, the rank-1 arrangements can easily be enumerated. The 36 possible arrangements of the rank- 1 tree include 6 of the form " $I I$ " that pair a crystal variant with itself and thus have no domain wall, 
representing rank-0 laminates, or single crystal variants. Another 6 contain pairings such as " 12 " that represent laminations of a crystal variant with another variant having the same spontaneous strain but opposite spontaneous polarization. In each case, a $180^{\circ}$ domain wall can form satisfying the exact compatibility conditions. These laminates are congruent, differing only by rotations. The remaining 24 laminates contain pairings such as "13" that represent laminations of one crystal variant with another having different spontaneous strain and polarization. Once again, the exact compatibility conditions can be met, this time by forming $90^{\circ}$ domain walls. These 24 laminates are again congruent by rotation. Effectively, then, there are just two families of rank-1 laminate in the polar tetragonal system, those with $90^{\circ}$ and those with $180^{\circ}$ domain walls. The notation $\{12\}$ and $\{13\}$ can be used to indicate these families.

Tsou et al. (2011) have similarly enumerated the rank-2 laminates in the polar tetragonal system. At first sight there are $6^{4}=1296$ arrangements. Removing those that either (i) represent lower rank laminations such as "1111", or (ii) cannot satisfy exact compatibility conditions ("1345" is an example of this, failing to satisfy (112)), or (iii) are rotations or reflections of some lower numbered pattern, leaves just eight distinct families. These are illustrated in figure 10 .

The domain walls shown using dashed lines in figure (10) are $180^{\circ}$ walls without a unique orientation. Consequently these walls can form wavy patterns, producing the "watermarks" commonly observed in ferroelectric microstructure. Figure (10) also shows notional domain walls where a crystal variant meets itself across an interface introduced by the laminate. In practice, no domain wall would form in these circumstances and the crystal would be continuous, without an interface. Upon checking for the presence of disclinations, it is found that only patterns $\{1112\},\{1221\}$, $\{1234\}$ and $\{1324\}$ are disclination free. These are zero-energy laminations within the constrained theory and are among the commonest patterns of domains observed in tetragonal ferroelectric crystals such as $\mathrm{BaTiO}_{3}$ ( Tsou et al. (2011); McGilly et al. (2010); Hooton and Merz (1955); Tagantsev et al. (2010)).

The polar tetragonal crystal structure is among the most important of the ferroelectric crystal systems, being found in $\mathrm{BaTiO}_{3}$ and in the PZT alloys $\mathrm{PbZr}_{x} \mathrm{TiO}_{(1-x)} \mathrm{O}_{3}$ as well as many others. The PZT alloy series also includes rhombohedral crystal structures, which have trigonal symmetry. 

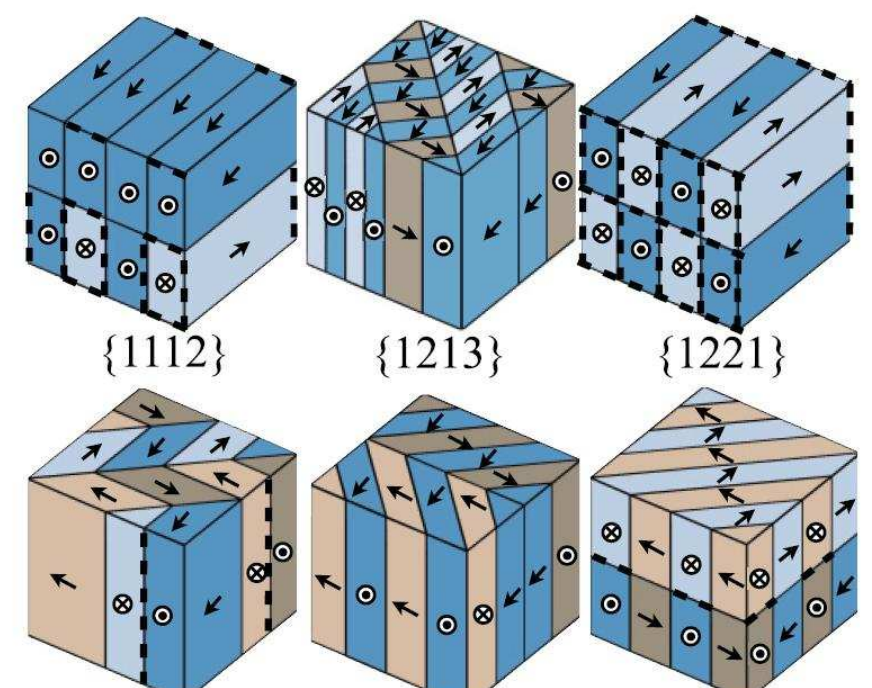

$\{1213\}$

$\{1221\}$

$\{1234\}$
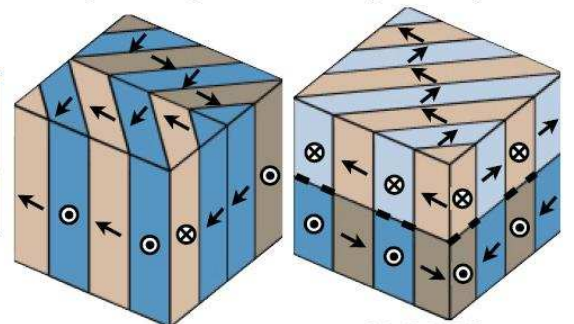

$\{1324\}$

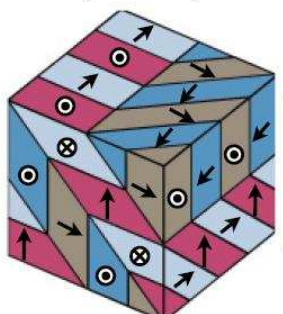

$\{1325\}$

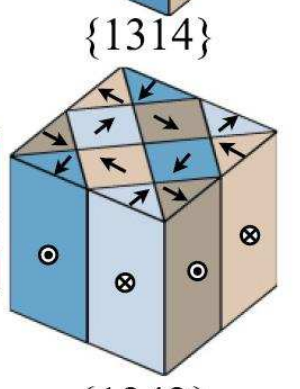

$\{1342\}$

Figure 10. The periodic repeating cells of the set of distinct rank-2 laminations in the tetragonal system.

The polar rhombohedral crystal system has eight variants as follows:

$$
\begin{aligned}
\boldsymbol{P}^{1}=\frac{P_{0}}{\sqrt{3}}\left(\begin{array}{c}
1 \\
1 \\
1
\end{array}\right) ; & \boldsymbol{P}^{2}=\frac{P_{0}}{\sqrt{3}}\left(\begin{array}{c}
-1 \\
-1 \\
-1
\end{array}\right) ; \\
\boldsymbol{P}^{3}=\frac{P_{0}}{\sqrt{3}}\left(\begin{array}{c}
-1 \\
1 \\
1
\end{array}\right) ; & \boldsymbol{P}^{4}=\frac{P_{0}}{\sqrt{3}}\left(\begin{array}{c}
1 \\
-1 \\
-1
\end{array}\right) ; \\
\boldsymbol{P}^{5}=\frac{P_{0}}{\sqrt{3}}\left(\begin{array}{c}
-1 \\
-1 \\
1
\end{array}\right) ; & \boldsymbol{P}^{6}=\frac{P_{0}}{\sqrt{3}}\left(\begin{array}{c}
1 \\
1 \\
-1
\end{array}\right) ; \\
\boldsymbol{P}^{7}=\frac{P_{0}}{\sqrt{3}}\left(\begin{array}{c}
1 \\
-1 \\
1
\end{array}\right) ; & \boldsymbol{P}^{8}=\frac{P_{0}}{\sqrt{3}}\left(\begin{array}{c}
-1 \\
1 \\
-1
\end{array}\right) ;
\end{aligned}
$$


with corresponding spontaneous strain states

$$
\begin{aligned}
\boldsymbol{\epsilon}^{1}=\boldsymbol{\epsilon}^{2} & =\epsilon_{0}\left(\begin{array}{ccc}
0 & 1 & 1 \\
1 & 0 & 1 \\
1 & 1 & 0
\end{array}\right) \\
\boldsymbol{\epsilon}^{3}=\boldsymbol{\epsilon}^{4} & =\epsilon_{0}\left(\begin{array}{ccc}
0 & -1 & -1 \\
-1 & 0 & 1 \\
-1 & 1 & 0
\end{array}\right) \\
\boldsymbol{\epsilon}^{5}=\boldsymbol{\epsilon}^{6} & =\epsilon_{0}\left(\begin{array}{ccc}
0 & 1 & -1 \\
1 & 0 & -1 \\
-1 & -1 & 0
\end{array}\right) \\
\boldsymbol{\epsilon}^{7}=\boldsymbol{\epsilon}^{8} & =\epsilon_{0}\left(\begin{array}{ccc}
0 & -1 & 1 \\
-1 & 0 & -1 \\
1 & -1 & 0
\end{array}\right)
\end{aligned}
$$

The crystal variants are pairwise compatible and from (93) and (94) it can be found that there are three distinct types of domain wall that can form. The corresponding rank- 1 laminates are $\{12\}$ with $180^{\circ}$ domain walls, $\{13\}$ with $70.5^{\circ}$ domain walls, and $\{14\}$ with $109.5^{\circ}$ domain walls. In this crystal system, equation (98) indicates that $\epsilon_{i j}^{r}=0$ whenever $i=j$ and so the macroscopic remanent strain includes only shear components in co-ordinates aligned with the crystallographic axes. Then (98)and (99) give:

$$
\left[\begin{array}{c}
\epsilon_{23}^{r} / \epsilon_{0} \\
\epsilon_{31}^{r} / \epsilon_{0} \\
\epsilon_{12}^{r} / \epsilon_{0} \\
\sqrt{3} P_{1}^{r} / P_{0} \\
\sqrt{3} P_{2}^{r} / P_{0} \\
\sqrt{3} P_{3}^{r} / P_{0} \\
1
\end{array}\right]=\left[\begin{array}{cccccccc}
1 & 1 & 1 & 1 & -1 & -1 & -1 & -1 \\
1 & 1 & -1 & -1 & -1 & -1 & 1 & 1 \\
1 & 1 & -1 & -1 & 1 & 1 & -1 & -1 \\
1 & -1 & -1 & 1 & -1 & 1 & 1 & -1 \\
1 & -1 & 1 & -1 & -1 & 1 & -1 & 1 \\
1 & -1 & 1 & -1 & 1 & -1 & 1 & -1 \\
1 & 1 & 1 & 1 & 1 & 1 & 1 & 1
\end{array}\right]\left[\begin{array}{l}
f^{1} \\
f^{2} \\
f^{3} \\
f^{4} \\
f^{5} \\
f^{6} \\
f^{7} \\
f^{8}
\end{array}\right]
$$

Noting once again that this has the form $\boldsymbol{e}=\boldsymbol{M} \boldsymbol{f}, \boldsymbol{M}$ is in this case a $7 \times 8$ matrix of rank 7. Thus there is a degree of freedom in the solution to (128), the general solution being

$$
\boldsymbol{f}=\boldsymbol{f}_{0}+\gamma\left[\begin{array}{lllllll}
1 & -1 & -1 & 1 & 1 & -1 & -1
\end{array}\right]^{T}
$$

where $\boldsymbol{f}_{0}$ is any particular solution for the set of volume fractions, and $\gamma$ is a scalar parameter that is limited in range by the requirement for all $f^{I} \geq 0$. Hence for any feasible macroscopic state of $\epsilon^{r}$ it is possible to find a set of volume fractions $f^{I}$ with at least one of the $f^{I}$ equal to zero. 
Averagely compatible laminates can be found for any $\epsilon^{r}$ in the convex hull of the set of $\boldsymbol{\epsilon}^{I}$, but these will typically have high rank and so are unlikely to form naturally. The macroscopic strain and polarization states that can be achieved with exactly compatible laminates have been explored by Tsou and Huber (2010). In considering the rank-2 exactly compatible laminates that can form, Tsou (2011) found the 14 families of laminates shown in figure 11 .

These rank-2 laminates have some remarkable properties. For example, the laminate designated "1458" can, through the choice of nodal volume fractions $f_{4}=f_{5}=f_{6}=f_{7}=1 / 4$, produce the "unpoled" macroscopic state $\boldsymbol{\epsilon}^{r}=0$. But by varying the volume fractions, a continuous range of laminates can be produced including both the "unpoled" state and a single variant state, representing a fully "poled" condition. Possibilities of this kind indicate the potential utility of the constrained theory in identifying low energy pathways that the domain patterns in ferroelectric crystals could follow, potentially enabling ferroelectric switching with very low coercive field. A discussion of the design of martensitic materials to achieve such low energy transformation paths has been given by Zhang and James (2009). In the case of ferroelectrics, the possibility of domain walls migrating through a laminate, transforming it continuously from one state of $\epsilon^{r}$ to another, provides a means to model the effect of applied macroscopic loads in the form of electric field and stress. The next section discusses the evolution of laminate domain patterns under applied loads.

\subsection{Evolution of laminate domain patterns}

A number of constitutive models for ferroelectric crystals have been developed around the concepts of compatible laminates (Yen et al. (2008); Weng and Wong (2009); Tsou et al. (2013)). The advantage of this approach, relative to the crystal plasticity methods described in section 4.1, is that some account is taken of the pattern of domains within a crystal, or within individual grains of a polycrystal. This is significant because the presence of particular arrangements of domains can affect the switching process. To illustrate models of this kind, a general model of ferroelectric switching based on the incremental motion of a known pattern of domain walls is first developed here. Following Huber and Cocks (2008), rate potentials are used to characterize the mobility of the domain walls.

Consider a region of a ferroelectric crystal occupying volume $V$, bounded by surface $S$, containing domains and domain walls such that the positions of the domain walls are defined by a set of $m$ configurational variables $a^{i}$, with $i=1 \ldots m$. As in the constrained theory, the domain walls are taken 


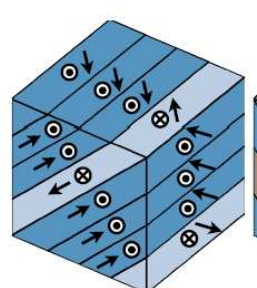

$\{1112\}$

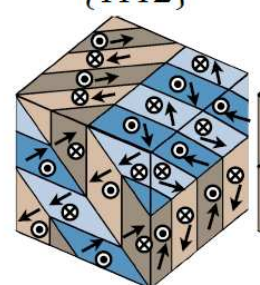

$\{1234\}$

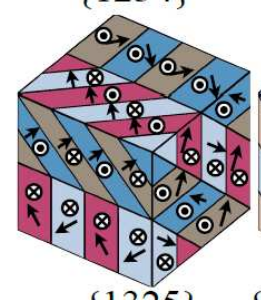

$\{1325\}$

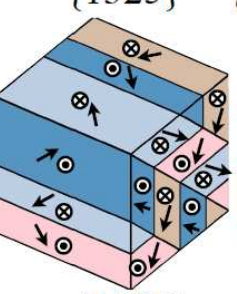

$\{1426$ \}

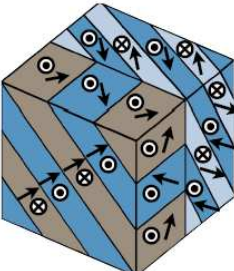

$\{1213\}$

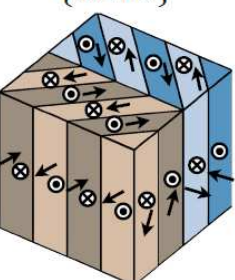

$\{1243\}$

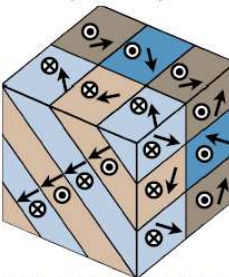

$\{1342\} /\{1432\}$

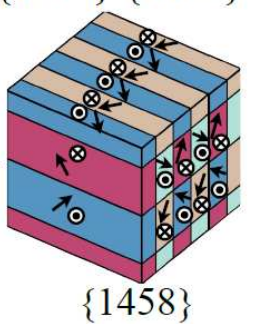

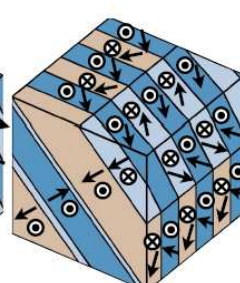

$\{1214\}$

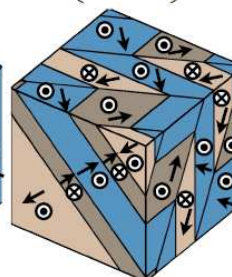

$\{1314\}$

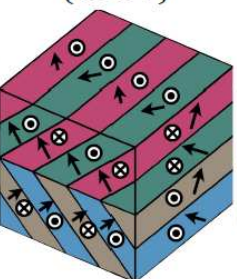

$\{1357\}$<smiles>[3H]C([3H])[3H]</smiles>

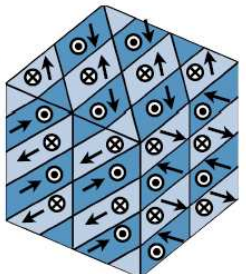

$\{1221\}$

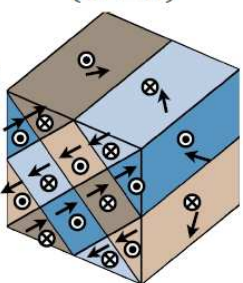

$\{1324\}$

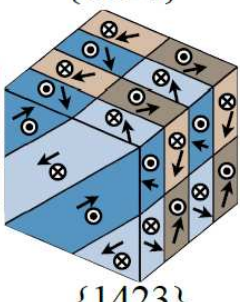

$\{1423\}$

Figure 11. The periodic repeating cells of the set of distinct rank-2 laminations in the rhombohedral system, after Tsou (2011). Arrows show the projections of each polarization vector onto the surface of the cell.

to be thin such that each material point lies within one of the domains, with spontaneous strain state $\epsilon_{i j}^{I}$ and spontaneous polarization $P_{i}^{I}$, corresponding to one of the $N$ crystal variants $(I \in 1 \ldots N)$. The domain walls themselves occupy area $A$. Let each material point experience local stress $\sigma_{i j}$ and electric field $E_{i}$. The Gibbs free energy of the system $G$ is the sum 
of an internal energy $U\left(\boldsymbol{\sigma}, \boldsymbol{E}, a^{i}\right)$ and potential energy, $\Omega$, due to boundary tractions $t_{i}$ and surface charge density $q$. The stored dielectric energy outside region $V$ is neglected here, but should be included whenever changes in external stored energy significantly influence the material behaviour. The Gibbs free energy in the current configuration may be written as

$$
G\left(\boldsymbol{\sigma}, \boldsymbol{E}, a^{i}\right)=\frac{1}{2} \int_{V} \sigma_{i j}\left(\epsilon_{i j}-\epsilon_{i j}^{I}\right)+E_{i}\left(D_{i}-P_{i}^{I}\right) d V-\int_{S} \phi q+t_{i} u_{i} d S
$$

where $I$ varies from point to point, indicating which crystal variant is present at each point.

A change in the configurational variables $\delta a^{i}$ causes points on the domain walls to displace by $\delta x$ resulting in a change $\delta G$ in the free energy. Define an equivalent thermodynamic pressure $F(v)$ on the domain walls, moving at speed $v$ such that

$$
\int_{A} F(v) \delta x d A=-\delta G
$$

$d A$ being an element of the domain wall area. Note that $F(v)$ can vary from point to point on the domain walls; also $G$ depends on the current configuration and loading, but is independent of $v$. Hence if the dependence of $F(v)$ on $v$ is specified, (131) ensures that the displacements $\delta x$ are energetically consistent with $\delta G$. Thus the motion of domain walls is driven by changes in $G$, but their increment of position is governed by $F(v)$. If the increment $\delta x$ happens over time increment $\delta t$, then in the limit $\delta t \rightarrow 0$

$$
\int_{A} F(v) v d A=-\dot{G}
$$

Now define a rate potential $\Psi\left(a^{i}, \dot{a}^{i}\right)$ as

$$
\Psi=\int_{A} \int_{0}^{v} F(\zeta) d \zeta d A
$$

A virtual variation $\delta \dot{a}^{i}$ in the rates of the configurational variables corresponds to variation $\delta v$ of the domain wall velocity at each point on the walls, and a variation $\delta \dot{G}$ in the rate of change of Gibbs free energy. Using (132) and (133)

$$
\delta \Psi=\int_{A} F(v) \delta v d A=-\delta \dot{G}
$$


Hence, defining a functional $\Pi$ by

$$
\Pi=\Psi+\dot{G}
$$

the rates $\dot{a}^{i}$ are such that $\Pi$ is stationary to variations $\delta \dot{a}^{i}$; that is

$$
\delta \Pi=0
$$

This variational principle provides a general framework for the thermodynamic evolution of configurational variables and similar methods have been widely applied in the study of microstructure evolution (Fischer et al. (2014)). In the present context, since $G$ is independent of the $\dot{a}^{i}$ then

$$
\dot{G}=\sum_{i} \frac{\partial G}{\partial a^{i}} \dot{a}^{i}
$$

or writing the $a^{i}$ as a row matrix $\boldsymbol{a}$,

$$
\dot{G}=\frac{\partial G}{\partial \boldsymbol{a}} \dot{\boldsymbol{a}}^{T}
$$

Then the variation in $\dot{G}$ is

$$
\delta \dot{G}=\frac{\partial G}{\partial \boldsymbol{a}} \delta \dot{\boldsymbol{a}}^{T}
$$

Application of (136) provides a set of equations for the $\dot{a}^{i}$, which can be coupled and non-linear. However, a particularly simple expression results if the system has linear kinetics, of the form

$$
\Psi=\frac{1}{2} \dot{\boldsymbol{a}} \boldsymbol{M} \dot{\boldsymbol{a}}^{T}
$$

where $M$ is a symmetric matrix of constant coefficients that defines the mobility of the domain walls. Then

$$
\delta \Psi=\dot{\boldsymbol{a}} \boldsymbol{M} \delta \dot{\boldsymbol{a}}^{T}
$$

and so

$$
-\frac{\partial G}{\partial \boldsymbol{a}} \delta \dot{\boldsymbol{a}}^{T}=\dot{\boldsymbol{a}} \boldsymbol{M} \delta \dot{\boldsymbol{a}}^{T}
$$

As this holds for any variation $\delta \dot{a}^{i}$ about the true rates $\dot{a}^{i}$, then the $\dot{a}^{i}$ can be found directly by

$$
\dot{\boldsymbol{a}}^{T}=-\boldsymbol{M}^{-1}\left(\frac{\partial G}{\partial \boldsymbol{a}}\right)^{T}
$$




\subsection{Example of domain pattern evolution}

The model of evolution of domain patterns outlined in section 5.5 can be illustrated by considering the evolution of a rank- 2 laminate under simple loading conditions. Take as an example a laminate in the family $\{1458\}$ of the polar rhombohedral crystal system. This family was shown in section 5.4 to allow a state of zero average remanent strain and polarization when the four crystal variants have equal volume fractions. These laminates also allow a fully polarized state when the volume fraction of any variant tends to unity. It can easily be shown that the $\{1458\}$ family is unique among rank2 laminates in allowing $\epsilon^{r}=0$. To see this, consider the general solution for the volume fractions of the crystal variants given in (129). When $\boldsymbol{\epsilon}^{r}=0$ the volume fractions take the form

$$
\boldsymbol{f}=\frac{1}{8}\left[\begin{array}{llllllll}
1 & 1 & 1 & 1 & 1 & 1 & 1 & 1
\end{array}\right]^{T}+\gamma\left[\begin{array}{lllllllll}
1 & -1 & -1 & 1 & 1 & -1 & -1 & 1
\end{array}\right]^{T}
$$

with $\gamma$ limited such that $0 \leq f^{I} \leq 1$. Setting $\gamma$ such that only four crystal variants are present gives

$$
\boldsymbol{f}=\frac{1}{4}\left[\begin{array}{llllllll}
1 & 0 & 0 & 1 & 1 & 0 & 0 & 1
\end{array}\right]^{T} \text { or } \boldsymbol{f}=\frac{1}{4}\left[\begin{array}{llllllll}
0 & 1 & 1 & 0 & 0 & 1 & 1 & 0
\end{array}\right]^{T}
$$

The first of these possibilities has only variant numbers $1,4,5$, and 8 present and so includes the $\{1458\}$ family. Since these four crystal variants produce a set of polarizations $\boldsymbol{P}^{I}$ with tetrahedral symmetry (see figure 12), it is evident that all permutations such as "1458", "1548", and so forth, are related by pure rotations or reflections. Similarly it can be seen that the second arrangement of volume fractions in (145) produces laminates such as " 2376 " which is a rotation of " 1458 ", and permutations thereof.

Now consider the evolution of the specific domain pattern "1458". A binary tree diagram for this laminate is shown in figure 13a. Application of the compatibility equations from section 5.3 shows that the domain wall orientations are given by

$$
\boldsymbol{n}^{1}=\left(\begin{array}{c}
0 \\
0 \\
1
\end{array}\right) ; \quad \boldsymbol{n}^{2}=\boldsymbol{n}^{3}=\left(\begin{array}{l}
1 \\
0 \\
0
\end{array}\right)
$$

The resulting pattern of domains is a rectangular grid or checkerboard as shown in cross-section in the $x-z$ plane in figure $13 \mathrm{~b}$. All of the internal interfaces are $109.5^{\circ}$ domain walls. For simplicity, let the material experience uniform stress and electric field with all components equal to zero except $\sigma_{11}$ and $E_{1}$. Also, let each crystal variant have identical material properties 


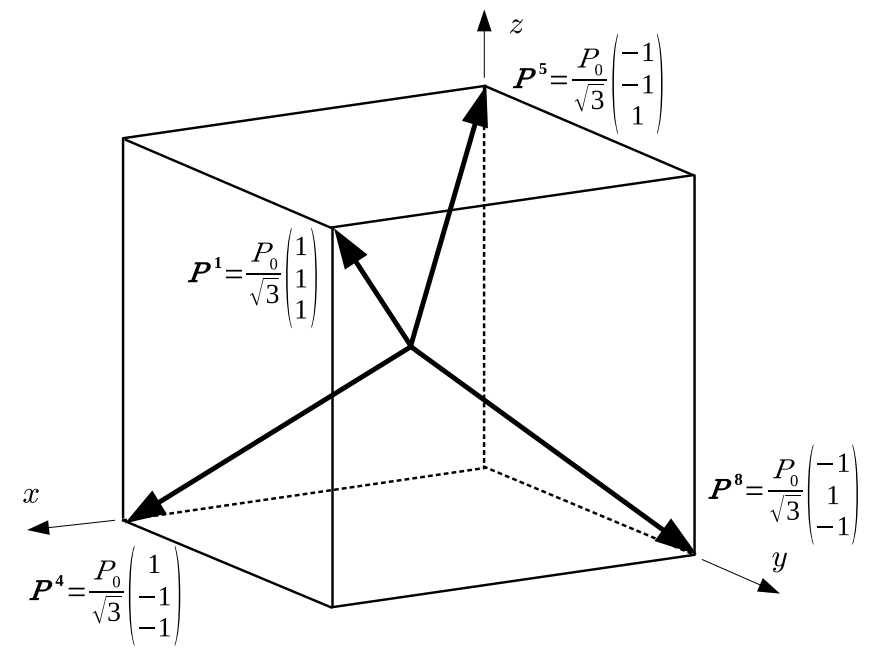

Figure 12. The polarization states of the four rhombohedral crystal variants in the laminate " 1458 ".

$s_{i j k l}^{E}$ and $\varepsilon_{i j}^{\sigma}$ in $x, y, z$ co-ordinates aligned with the crystallographic axes. Define the positions of the domain walls within a unit volume of the material by two configurational variables $\left(a^{1}, a^{2}\right)$ as shown in figure $13 \mathrm{~b}$, such that the volume fractions of the individual crystal variants are:

$$
f^{1}=a^{1} a^{2} ; \quad f^{4}=a^{1}\left(1-a^{2}\right) ; \quad f^{5}=a^{2}\left(1-a^{1}\right) ; \quad f^{8}=\left(1-a^{1}\right)\left(1-a^{2}\right)
$$

In order to find $\partial G / \partial \boldsymbol{a}$, recall that $G=U+\Omega$, and note that for the specified loading with only $\sigma_{11}$ and $E_{1}$ the internal energy $U$ is given by

$$
U=\int_{V} \frac{1}{2} \sigma_{11} s_{1111}^{E} \sigma_{11}+\frac{1}{2} E_{1} \varepsilon_{11}^{\sigma} E_{1}+E_{1} d_{111} \sigma_{11} d V
$$

while the potential energy of boundary tractions and charges is

$$
\begin{aligned}
\Omega= & -\int_{S} \phi q+t_{i} u_{i} d S \\
= & -\int_{V} \sigma_{11} s_{1111}^{E} \sigma_{11}+E_{1} \varepsilon_{11}^{\sigma} E_{1}+2 E_{1} d_{111} \sigma_{11} \\
& \quad+E_{1} P_{1}^{I}+\sigma_{11} \epsilon_{11}^{I} d V
\end{aligned}
$$


(a)

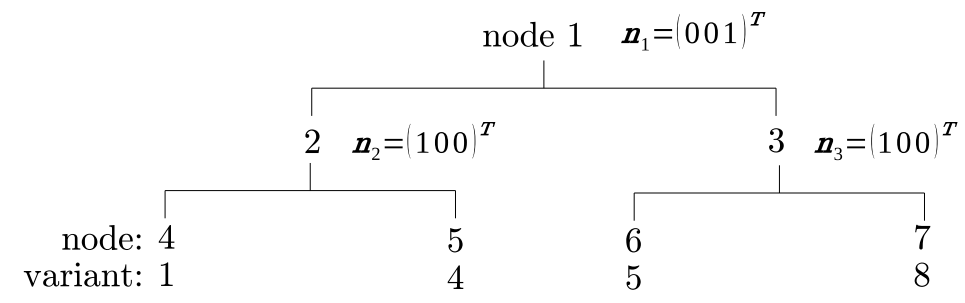

(b)

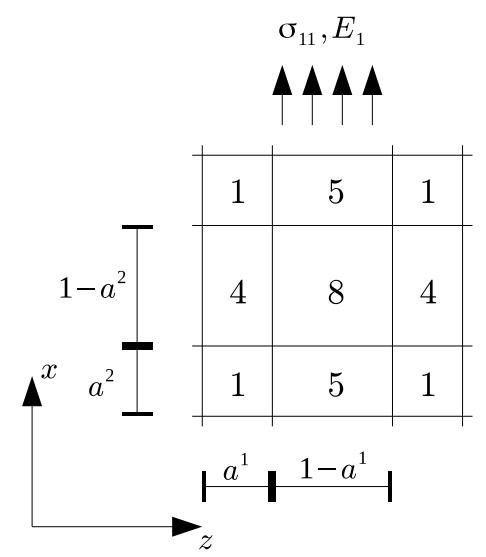

Figure 13. (a) Binary tree diagram for the laminate "1458", and (b) arrangement of crystal variants in the $x-z$ plane.

where use has been made of Gauss' theorem and electromechanical equilibrium to convert to a volume integral. The simplifying assumptions of uniform $s_{i j k l}^{E}$ and $\varepsilon_{i j}^{\sigma}$ imply that the corresponding terms in (148) and (149) will not enter into the expression for $\partial G / \partial \boldsymbol{a}$. However, it is expected that the piezoelectric coefficient $d_{111}$ will differ among the variants because of their differing polar axes. From symmetry, it can be seen that the $d_{111}$ coefficient will be identical in variants 1 and 4, and have an opposite (negative) value in variants 5 and 8 . Let variants 1 and 4 have $d_{111}=d_{0}$ and variants 5 and 8 have $d_{111}=-d_{0}$. Also, $\epsilon_{11}^{I}=0$ for all four variants. Then, for a unit volume of the material, using (147)-(149):

$$
\frac{\partial G}{\partial a^{1}}=-2 E_{1}\left(\sigma_{11} d_{0}+\frac{P_{0}}{\sqrt{3}}\right)
$$


and

$$
\frac{\partial G}{\partial a^{2}}=0
$$

The uniaxial loading case considered here does not drive changes in the second degree of freedom, as indicated by (151). This is as expected from the symmetry of the loading and the crystal variants. The rate $\dot{a}^{1}$ is then specified by rate potential $\Psi$. Taking the simple case of linear kinetics, given by

$$
\Psi=\frac{1}{2}\left(\begin{array}{ll}
\dot{a}^{1} & \dot{a}^{2}
\end{array}\right)\left(\begin{array}{cc}
m & 0 \\
0 & m
\end{array}\right)\left(\begin{array}{c}
\dot{a}^{1} \\
\dot{a}^{2}
\end{array}\right)
$$

per unit area, the evolution of domains is governed by

$$
\dot{a}^{1}=\frac{2 E_{1}}{m}\left(\sigma_{11} d_{0}+\frac{P_{0}}{\sqrt{3}}\right)
$$

The evolution of the laminate can be simulated by integrating (153), using (147) and (98) to reconstruct the remanent strain and polarization state. Additionally, the constraint $0 \leq a^{i} \leq 1$ must be enforced. Figure 14 shows the polarization response of the laminate when subjected to sinusoidal loading $E_{1}=E_{0} \sin (\omega t)$ with $\omega=2 \pi \mathrm{rad} \mathrm{s}^{-1}$ and $E_{0} P_{0} / m=30$, starting from a state with $a^{1}=0.5$. The response is shown with no stress and with a compressive stress $\sigma_{11}=-0.2 P_{0} / d_{0}$, which reduces the rate of ferroelectric switching through piezoelectric coupling.

Other examples have been given by Huber and Cocks (2008) and Tsou et al. (2013).

\section{$6 \quad$ Summary and Outlook}

This chapter has reviewed a variety of methods for modelling the electromechanical response of ferroelectrics. The methods are derived from classical theories of plasticity, energy minimization, and the use of thermodynamic principles that have wide application in mechanics. It is expected that extensions of these methods can also provide insight into other ferroic materials and multiferroics. While the chapter makes a general progression from macroscopic towards microscopic models, it does not address finer scale modelling methods such as Ginzburg-Landau phase field models, molecular dynamics or atomistics, each of which has made a substantial contribution to the understanding of ferroic materials. At present the fine-scale models are restricted by the limits of practical computation to regions of material of size in the order of nanometres. However, coupling of fine-scale to coarsescale is an ongoing endeavor that has already made substantial progress. 


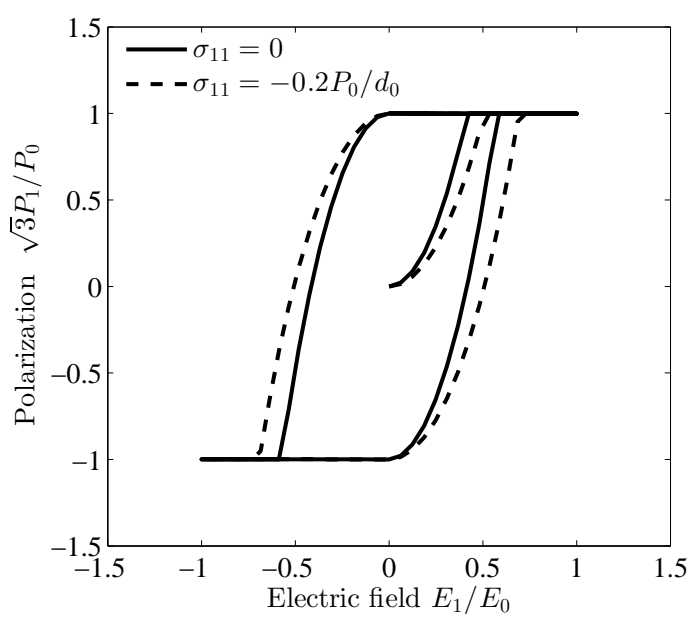

Figure 14. Polarization response of the "1458" laminate.

With continued growth in the applications of and the demand for ferroic materials, it can be expected that models of ferroelectrics and related materials will continue to provide a rich source of insight for engineering design.

\section{Acknowledgements}

Thanks are due to Nien-Ti Tsou for preparing and allowing the use of figures showing domain patterns, as well as for many helpful and informative discussions.

\section{Bibliography}

Arlt, G., Twinning in ferroelectric and ferroelastic ceramics: stress relief, Journal of Materials Science, 25:2655-2666,1990.

Arlt, G. and Sasko, P., Domain configuration and equilibrium size of domains in $\mathrm{BaTiO}_{3}$ ceramics, Journal of Applied Physics, 51:49564960,1980 .

Ball, J.M. and James, R.D., Fine phase mixtures as minimizers of energy, Archive for Rational Mechanics and Analysis, 11:13-52, 1987.

Bassiouny, E., Ghaleb, A.F. and Maugin, G.A., Thermodynamical formulation for coupled electromechanical hysteresis effects. 1. Basic equations, International Journal of Engineering Science, 26:1279-1295, 1988. 
Bhattacharya, K., Comparison of the geometrically nonlinear and linear theories of martensitic transformtion, Continuum Mechanics and Thermodynamics, 5:205-242,1993.

Bhattacharya, K., Microstructure of Martensite, Why it forms and how it gives rise to the shape-memory effect, Oxford University Press, 2003

Bisegna, P. and Luciano, R., Variational bounds for the overall properties of piezoelectric composites, Journal of the Mechanics and Physics of Solids, 44:583-602, 1996.

Burcsu, E., Ravichandran, G. and Bhattacharya, K., Large electrostrictive actuation of barium titanate single crystals, Journal of the Mechanics and Physics of Solids, 52:823-846, 2004.

Cocks, A.C.F. and McMeeking, R.M., A phenomenological constitutive law for the behaviour of ferroelectric ceramics, Ferroelectrics, 228:219-228, 1999.

Dayal, K. and Bhattacharya, K., A real-space non-local phase-field model of ferroelectric domain patterns in complex geometries, Acta Materialia, 55:1907-1917,2007.

Devonshire, A.F., Theory of Barium Titanate. 1., Philosophical Magazine, 40:1040-1063, 1949.

Dunn, M.L. and Taya, M., Micromechanics predictions of the effective electroelastic mouli of piezoelectric composites, International Journal of Solids and Structures, 30:161-175, 1993.

Eshelby, J.D., The detrmination of the elastic field of an ellipsoidal inclusion, and related problems, Proceedings of the Royal Society of London A, 241:376-396, 1957.

Fischer, F.D., Svoboda, J. and Petryk, H., Thermodynamic extremal principles for irreversible processes in materials science, Acta Materialia, 67:120, 2014.

Hall, D.A., Steuwer, A., Cherdhirunkorn, B., Withers, P. and Mori, T., Micromechanics of residula stress and texture development due to poling in polycrystalline ferroelectric ceramics, Journal of the Mechanics and Physics of Solids, 53:249-260,2005.

Haug, A., Huber, J.E., Onck, P.R. and Van der Giessen, E., Multigrain analysis versus self-consistent estimates of ferroelectric polycrystals, Journal of the Mechanics and Physics of Solids, 55:648-665,2007.

Hill, R., A self-consistent mechanics of composite materials, Journal of the Mechanics and Physics of Solids, 13:213-222, 1965.

Hooton, J.A. and Merz, W.J., Etch patterns and ferroelectric domains in $\mathrm{BaTiO}_{3}$ single crystals, Physical Review, 98:409-413, 1955.

Huber, J.E. and Cocks, A.C.F., A variational model of ferroelectric microstructure, Proceedings of ASME SMASIS08, Ellicott City, MD, USA 28-30 October, 2008. 
Huber, J.E. and Fleck, N.A., Multi-axial electrical switching of a ferroelectric: teory versus experiment, Journal of the Mechanics and Physics of Solids, 49:785-811, 2001.

Huber, J.E. and Fleck, N.A., Ferroelectric switching: a micromechanics model versus measured behaviour, European Journal of Mechanics ASolids, 23:203-217, 2004.

Huber, J.E., Fleck, N.A., Landis, C.M. and McMeeking, R.M., A constitutive model for ferroelectric polycrystals, Journal of the Mechanics and Physics of Solids, 47:1663-1697, 1999.

Hwang, S.C., Lynch, C.S. and McMeeking, R.M., Ferroelectric/ferroelastic interactons and a polarization switching model, Acta Metallurgica et $\mathrm{Ma}$ terialia, 43:2073-2084, 1995.

James, R.D. and Hane, K.F., Martensitic transformations and shapememory materials, Acta Materialia, 48:197-222,2000.

Jones, J.L., Slamovich, E.B. and Bowman, K.J., Domain texture distributions in tetrgonal lead zirconate titanate by X-ray and neutron diffraction, Journal of Applied Physics, 97:034113,2005.

Kamlah, M., Liskowsky, A.C., McMeeking, R.M. and Balke, H., Finite element simulation of a polycrystalline ferroelectric based on a multidomain single crystal switching model, International Journal of Solids and Structures, 42:2949-2964,2005.

Kamlah M.,and Tsakmakis, C., Phenomenological modelling of the nonlinear electromechanical coupling in ferroelectrics, International Journal of Solids and Structures, 36:669-695, 1999.

Kanoute, P., Boso, D.P., Chaboche, J.L. and Schrefler, B.A., Multiscale methods for composites: A review, Archives of Computational Methods in Engineering, 16:31-75, 2001.

Kouznetsova, V., Brekelmans, W.A.M. and Baaijens, F.P.T., An approach to micro-macro modeling of heterogeneous materials, Computational $\mathrm{Me}$ chanics, 27:37-48, 2001.

Landis, C.M., Fully coupled, multi-axial, symmetric constitutive laws for polycrystalline ferroelectric ceramics, Journal of the Mechanics and Physics of Solids, 50:127-152, 2002.

Li, J.Y. and Liu, D., On ferroelectric crystals with engineered domain configurations, Journal of the Mechanics and Physics of Solids, 52:17191742,2004

Li, Y.L., Hu, S.Y., Liu, Z.K. and Chen L.Q., Phase-field model of domain structures in ferroelectric thin films, Applied Physics Letters, 78:38783880, 2001.

Lines, M.E. and Glass, A.M., Principles and Applications of Ferroelectrics and Related Materials, Oxford University Press, 1977. 
Liu, Q.D. and Huber, J.E., State dependent linear moduli in ferroelectrics, International Journal of Solids and Structures, 44:5635-5650, 2007.

Liu, T. and Lynch, C. S., Domain engineered relaxor ferroelectric single crystals, Continuum Mechanics and Thermodynamics, 18:119-135, 2006.

Loge, R.E. and Suo, Z., Nonequilibrium thermodynamics of ferroelectric domain evolution, Acta Materialia, 44:3429-3438, 1996.

Lynch, C.S., The effect of uniaxial stress on the electro-mechanical response of 8/65/35 PLZT, Acta Materialia, 44:4138-4148, 1996.

McGilly, L.J., Schilling, A. and Gregg, J.M., Domain bundle boundaries in single crystal $\mathrm{BaTiO}_{3}$ lamellae: searching for naturally forming dipole flux-closure/quadrupole chains, Nano Letters, 10:4200-4205, 2010.

Park, S.E. and Shrout, T.R., Ultrahigh strain and piezoelectric behaviour in relaxor based ferroelectric single crystals, Journal of Applied Physics, 82:1804-1811,2007.

Pathak, A. and McMeeking, R.M., Three-dimensional finite element simulations of ferroelectric poycrystals inder electrical and mechanical loading, Journal of the Mechanics and Physics of Solids, 56:663-683,2008.

Shu, Y.C. and Bhattacharya, K., Domain patterns and macroscopic behaviour of ferroelectric materials, Philosophical Magazine B, 81:20212054, 1987.

Sidorkin, A.S., Domain Structure in Ferroelectric and Related Materials, Cambridge International Science Publishing, 2006.

$\mathrm{Su}, \mathrm{Y}$. and Landis, C.M., Continuum thermodynamics of ferroelectric domain evolution: Theory, finite element implementation, and application to domin wall pinning, Journal of the Mechanics and Physics of Solids, 55:280-305, 2004.

Tangantsev, A.K., Cross, L.E. and Fousek, J., Domains in Ferroic Crystals and Thin Films, Springer, New York, 2010.

Tsou, N.T. Compatible domain structures in ferroelectric single crystals, PhD Thesis, Univ. of Oxford, 2011.

Tsou, N.T. and Huber, J.E., Compatible domain structures and the poling of single crystal ferroelectrics, Mechanics of Materials, 42:740-753,2010.

Tsou, N.T., Huber, J.E. and Cocks, A.C.F., Evolution of compatible laminate domain structures in ferroelectric single crystals, Acta Materialia, 56:2117-2135, 2008.

Tsou, N.T., Potnis, P.R. and Huber, J.E., Classification of laminate domain patterns in ferroelectrics, Physical Review B, 83:184120,2011.

Völker, B. and Kamlah, M., Large-signal analysis of typical ferroelectric domain structures using phase-field modeling, Smart Materials and Structures, 21:055013, 2012. 
Wang, J.J., Ma, X.Q., Li, Q., Britson, J. and Chen, L.Q., Phase-transitions and domain structures of ferroelectric nanoparticales: Phase-field model incorporating strong elastic and dielectric inhomogeneity, Acta Materialia, 61:7591-7603, 2013.

Wang, J., Kamlah, M. and Zhang, T.-Y., Phase field simulations of ferroelectric nanoparticales with different long-range-electrostatic and -elastic interactions Journal of Applied Physics, 105:014104, 2009.

Wang, J., Shi, S.Q., Chen L.Q., Li, Y.L. and Zhang, T.Y. Phase-field simulations of ferroelectric/ferroelastic polarization switching, Acta Materialia, 52:749-764, 2004.

Weng, G.J. and Wong, D.T., Thermodynamic driving force in ferroelectric crystals with a rank-2 laminated domain pattern,and a study of enhanced electrostriction, Journal of the Mechanics and Physics of Solids, 57:571$597,2009$.

Wu, T., Zhao, P., Bao, M.Q., Bur, A., Hockel, J.L., Wong, K., Mohanchandra, K.P., Lynch, C.S. and Carman, G.P., Domain engineered switchable strain states in ferroelectric $(011)\left[\mathrm{Pb}\left(\mathrm{Mg}_{1 / 3} \mathrm{Nb}_{2 / 3} \mathrm{O}_{3}\right]_{(1-x)^{-}}\right.$ $\left[\mathrm{PbTiO}_{3}\right]_{(x)}(\mathrm{PMN}-\mathrm{PT}, x \approx 0.32)$ single crystals, Journal of Applied Physics, 109:124101,2011.

$\mathrm{Xu}$, Y., Ferroelectric Materials and their Applications, North Holland Press, 1991.

Yen, J.H., Shu, Y.C., Shieh, J. and Yeh, J.H., A study of electromechanical switching in ferroelectric single crystals. Journal of the Mechanics and Physics of Solids, 56:2117-2135, 2008.

Zhang, Z.Y., James, R.D. and Muller, S., Energy barriers and hysteresis in martensitic phase transformations, Acta Materialia, 57:4332-4352,2009. 